\title{
Histone deacetylase inhibitor panobinostat induces calcineurin degradation in multiple myeloma
}

\author{
Yoichi Imai, ${ }^{1}$ Eri Ohta, ${ }^{1}$ Shu Takeda, ${ }^{2}$ Satoko Sunamura, ${ }^{2}$ Mariko Ishibashi, ${ }^{3}$ Hideto Tamura, ${ }^{3}$ \\ Yan-hua Wang, ${ }^{1}$ Atsuko Deguchi, ${ }^{4}$ Junji Tanaka, ${ }^{1}$ Yoshiro Maru, ${ }^{4}$ and Toshiko Motoji \\ 'Department of Hematology, Tokyo Women's Medical University, Tokyo, Japan. ²Department of Physiology and \\ Cell Biology, Graduate School of Medicine and Dental Sciences, Tokyo Medical and Dental University, Tokyo, Japan. \\ ${ }^{3}$ Division of Hematology, Department of Medicine, Nippon Medical School, Tokyo, Japan. \\ ${ }^{4}$ Department of Pharmacology, Tokyo Women's Medical University, Tokyo, Japan.
}

Multiple myeloma (MM) is a relapsed and refractory disease, one that highlights the need for developing new molecular therapies for overcoming of drug resistance. Addition of panobinostat, a histone deacetylase (HDAC) inhibitor, to bortezomib and dexamethasone improved progressionfree survival (PFS) in relapsed and refractory MM patients. Here, we demonstrate how calcineurin, when inhibited by immunosuppressive drugs like FK506, is involved in myeloma cell growth and targeted by panobinostat. mRNA expression of PPP3CA, a catalytic subunit of calcineurin, was high in advanced patients. Panobinostat degraded PPP3CA, a degradation that should have been induced by inhibition of the chaperone function of heat shock protein 90 (HSP90). Cotreatment with HDAC inhibitors and FK506 led to an enhanced antimyeloma effect with a greater PPP3CA reduction compared with HDAC inhibitors alone both in vitro and in vivo. In addition, this combination treatment efficiently blocked osteoclast formation, which results in osteolytic lesions. The poor response and short PFS duration observed in the bortezomib-containing therapies of patients with high PPP3CA suggested its relevance to bortezomib resistance. Moreover, bortezomib and HDAC inhibitors synergistically suppressed MM cell viability through PPP3CA inhibition. Our findings underscore the usefulness of calcineurin-targeted therapy in MM patients, including patients who are resistant to bortezomib.

Authorship note: Y. Imai and E. Ohta contributed equally to this work.

Conflict of interest: The authors have declared that no conflict of interest exists.

Submitted: October 9, 2015 Accepted: March 22, 2016 Published: April 21, 2016

Reference information: JCl Insight. 2016;1(5):e85061. doi:10.1172/jci.insight.85061.

\section{Introduction}

Multiple myeloma (MM) is an incurable hematologic malignancy. As MM is prone to relapse, it is a type of cancer that could acquire resistance to many chemotherapeutic drugs including bortezomib, a proteasome inhibitor. Combined treatment with bortezomib and panobinostat has proven effective in patients, including those previously treated with bortezomib $(1,2)$. Panobinostat was recently approved by the FDA for relapsed and refractory patients who have previously undergone treatments including bortezomib. Proteasome and histone deacetylase (HDAC) inhibitors have been postulated to block aggresome formations, thereby inhibiting the proliferation of malignant cells (3-8). Recently, HDAC6-independent induction of apoptosis via ROS generation following treatment with proteasome and HDAC inhibitors was reported (9). However, it remains to be elucidated which pathogenetic molecules in MM cells would be targeted by this combination therapy. Here, we investigated which potential molecules promoting MM progression would be targeted by proteasome and HDAC inhibitors.

Calcineurin is a calcium-calmodulin-dependent serine/threonine protein phosphatase that plays a critical role in T cell activation following T cell receptor engagement. Calcineurin inhibitors such as FK506 and cyclosporine A are widely used in clinical practice as immunosuppressive drugs (10). Calcineurin activation has previously been reported to play a critical role in the pathogenesis of hematological malignancies in $\mathrm{T}$ cell acute lymphoblastic leukemia (T-ALL) (11). Although calcineurin has been studied mainly in T cells, a few reports point to its importance in B cells. One study showed that decreased calcineurin activation caused defective B cell activation (12). The results suggest that calcineurin activation might be important to the pathogenesis of B cell malignancies, including MM. In fact, the nuclear factor of activated $\mathrm{T}$ cells, cytoplasmic, calcineurin-dependent 1 (NFATc1), a dephosphorylation target of calcineurin, is activated and 
A

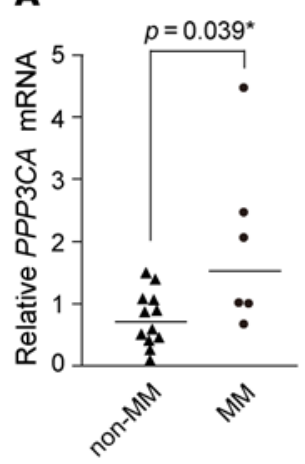

B

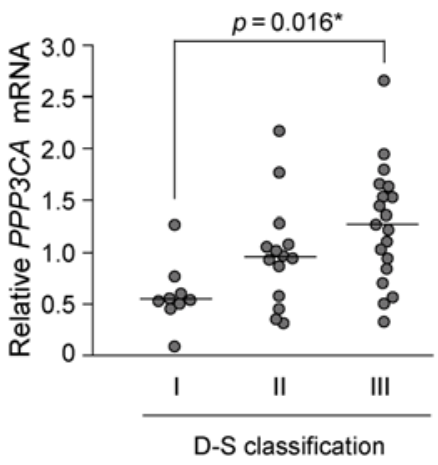

C

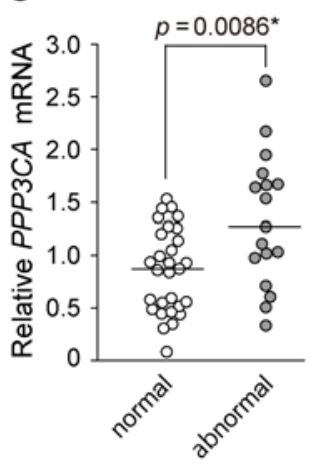

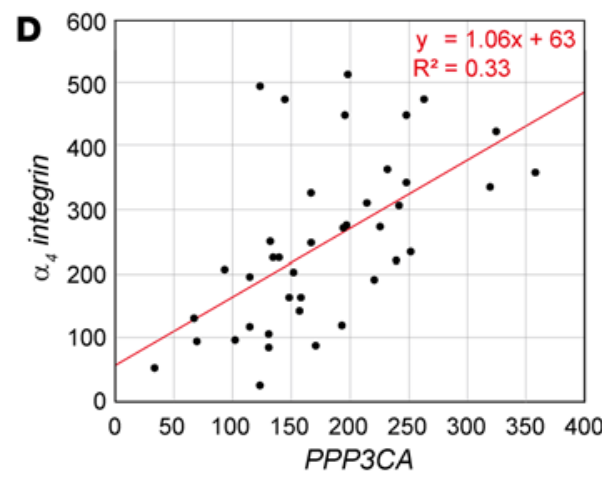

Figure 1. High expression of PPP3CA (protein phosphatase 3, catalytic subunit, an isozyme) mRNA in advanced MM (multiple myeloma) mRNA in advanced MM patients. (A) PPP3CA mRNA expression in non-MM $(n=12)$ and MM cell lines $(n=6)$ is displayed. Non-MM cell lines are as follows. Acute myeloid leukemia-derived: HEL, HL60, KG-1, THP-1, and U937; B cell acute lymphoblastic leukemia: BALL-1 and NALM6; T cell acute lymphoblastic leukemia (T-ALL): Jurkat and Molt-4; chronic myelogenous leukemia: K562; and B cell lymphoma: Daudi and Raji. MM cell lines are as follows: U266, KMS-11, KMS-12PE, KMS-18, KMS-26, and RPMI8226. Horizontal line, median. Difference between 2 groups was analyzed using 1-tailed $t$ test. *, significant. Two biologically independent experiments were performed. (B) PPP3CA mRNA expression in MM patients with stage I $(n=9)$, II $(n=14)$, or III $(n=19)$ is displayed. D-S, Duri-Salmon classification. Horizontal line, median. The differences between 3 groups of samples was analyzed by ANOVA by the 1-way layout. When the statistic model proved significant, the differences between combinations of the 2 groups were analyzed using a Tukey-Kramer test for multiple comparisons.*, significant. (C) PPP3CA mRNA expression in MM patients with normal $(n=29)$ or abnormal $(n=17)$ serum LDH is displayed. Horizontal line, median. Difference between 2 groups was analyzed using 1-tailed $t$ test. ${ }^{*}$, significant. (D) mRNA expression of PPP3CA and $\alpha_{4}$ integrins in samples from MM patients reported in the study of Agnelli (20). Correlation coefficient between PPP3CA and $\alpha_{4}$ integrins expression is displayed.

promotes proliferation and cell survival in diffuse large B cell lymphoma-derived cell lines $(13,14)$. When we investigated whether calcineurin activation would affect MM cell survival, we found that PPP3CA (protein phosphatase 3, catalytic subunit, $\alpha$ isozyme), its catalytic subunit, is involved in myeloma cell growth.

Heat shock protein 90 (HSP90) functions as a chaperone stabilizing its client proteins and, as a nonhistone protein, is one of the targets of HDAC inhibition (15). The chaperone function is inhibited by the acetylation induced by HDAC inhibitors (16). Panobinostat induces hyperacetylation of HSP90 in acute myeloid leukemia cells and inhibits its chaperone function, thereby leading to the proteasomal degradation of client proteins such as CXCR4 and AML1/ETO9a, both of which are involved in leukemogenesis (17, 18). These results would suggest that the ability of HDAC inhibitors to block the chaperone function of HSP90 might be important for facilitating their anticancer effects. We have shown that HDAC inhibition leads to the protein degradation of PPP3CA. Furthermore, we have demonstrated that PPP3CA was the common target of bortezomib and HDAC inhibitors and that aberrantly enhanced PPP $3 C A$ expression promoted bortezomib resistance. Our study points to the new role of calcineurin in the pathogenesis of MM and thus supports the possibility of developing novel strategies to target PPP3CA in MM patients.

\section{Results}

Aberrantly increased PPP $3 C A$ expression observed in MM cell lines and MM cells isolated from patients with advanced disease. To study the potentially pathogenic role of PPP3CA in MM, we examined the expression levels of $P P P 3 C A$ in $\mathrm{MM}$ patients. We compared PPP3CA expression of $\mathrm{MM}$ cells isolated from patients suffering different stages of MM. A previous study involved high-resolution genomic and mRNA expression profiling of MM cells, thereby revealing candidate oncogenes for MM (19). We examined the expression of 179 candidate genes in MM patients previously reported using the Gene Expression Omnibus (GEO; http:// www.ncbi.nlm.nih.gov/geo/) database (20), comparing it among different stages of patients. The disease stages of each patient were determined using the Durie-Salmon staging classification system (21). Those genes highly expressed in most advanced stage III cases (21) are likely to be involved in MM progression. Among the candidate genes examined, $P P P 3 C A$ was one of 8 genes whose mRNA expression was significantly higher in samples from stage III patients compared with those from stage I patients (Supplemental Figure 1; supplemental material available online with this article; doi:10.1172/jci.insight.85061DS1).

We analyzed PPP3CA expression in non-MM and MM cell lines and found high PPP3CA expression only in the latter (Figure 1A). We then examined our MM patient samples (Supplemental Table 1). We found higher expression of $P P P 3 C A$ in stage III compared with stage I (Figure 1B). Among the clinical 
A

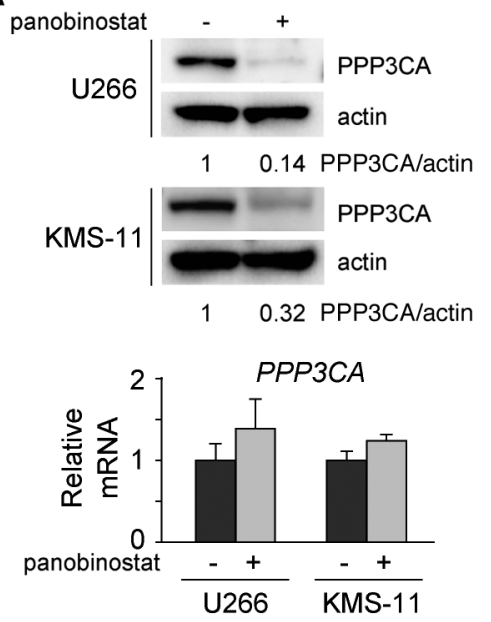

$\mathbf{F}$

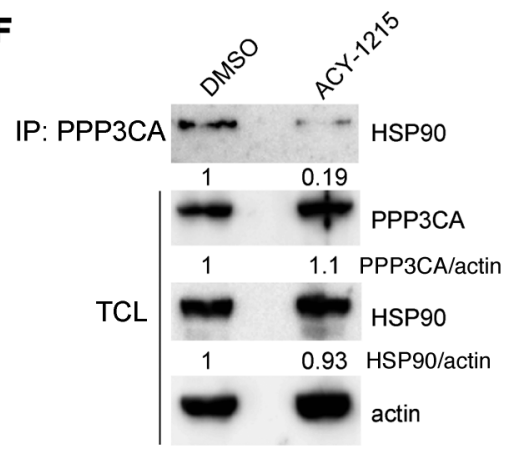

B
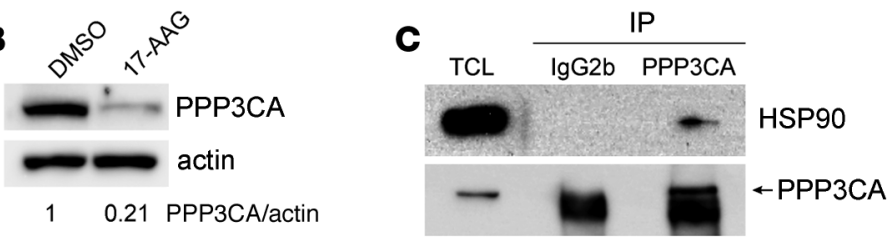

D

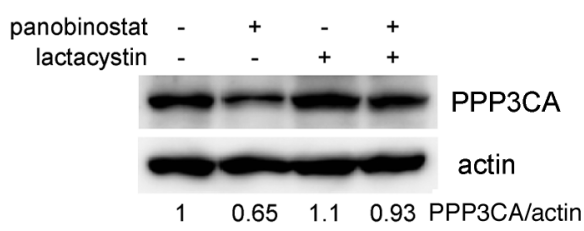

E

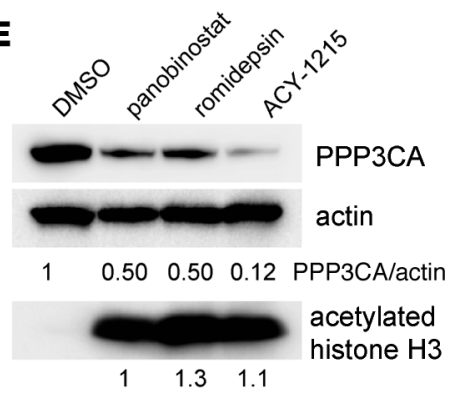

G

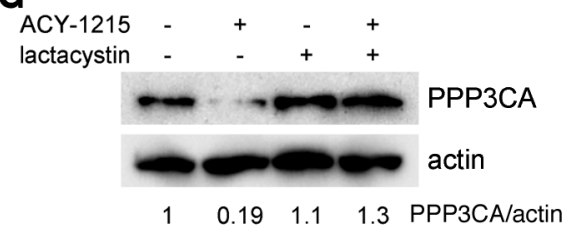

H

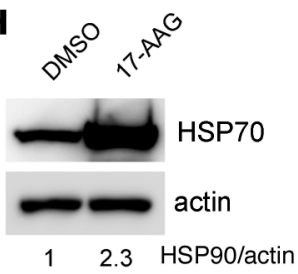

\section{I}

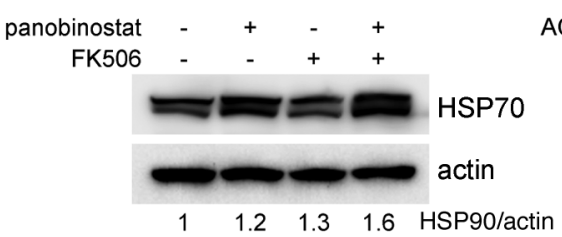

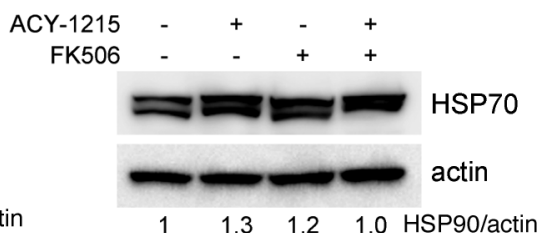

Figure 2. PPP3CA protein is degraded by HDAC (histone deacetylase) inhibitors in MM cells. The degree of protein expression change estimated by quantitative analyses of bands is displayed as indicated. (A) U266 and KMS-11 were treated with $20 \mathrm{nM}$ panobinostat for $48 \mathrm{~h}$ followed by Western blot analysis. Actin served as a loading control. Nine (U266) and 3 (KMS-11) biologically independent experiments were performed. To determine the expression of PPP3CA mRNA in treated cells for $24 \mathrm{~h}$, we performed relative quantification real-time PCR $(n=6)$. Four (U266) and 2 (KMS-11) biologically independent experiments were performed. (B) PPP3CA expression in U266 treated with DMSO (vehicle control) or $600 \mathrm{nM} 17-\mathrm{AAC}$ for $24 \mathrm{~h}$. Three biologically independent experiments were performed. (C) IP assays using IgG2b or anti-PPP3CA antibody in U266. TCL, total cell lysate. Five biologically independent experiments were performed. (D) Protein expression in U266 treated with $20 \mathrm{nM}$ panobinostat, $5 \mathrm{nM}$ lactacystin, or both panobinostat and lactacystin for $48 \mathrm{~h}$. Two biologically independent experiments were performed. (E) Protein expression in U266 treated with $20 \mathrm{nM}$ panobinostat, $2 \mathrm{nM}$ romidepsin, or $2 \mu \mathrm{M}$ ACY-1215 for $48 \mathrm{~h}$. Two biologically independent experiments were performed. (F) IP assays using anti-PPP3CA antibody in U266 treated with $2 \mu \mathrm{M}$ ACY-1215 for $16 \mathrm{~h}$. Two biologically independent experiments were performed. (C) Protein expression in U266 treated with $2 \mu \mathrm{M}$ ACY-1215, $5 \mathrm{nM}$ lactacystin, or both ACY-1215 and lactacystin for $48 \mathrm{~h}$. Two biologically independent experiments were performed. (H) HSP70 protein expression in U266 treated with $600 \mathrm{nM} 17-\mathrm{AAC}$ for 24 hours. Two technically independent experiments were performed. (I) HSP70 expression in U266 treated with $20 \mathrm{nM}$ panobinostat, 10 $\mu \mathrm{M}$ FK506, or both panobinostat and FK506 as indicated for $36 \mathrm{~h}$. Cotreatment with $0.75 \mu \mathrm{M}$ ACY-1215 and $10 \mu \mathrm{M}$ FK506 for $48 \mathrm{~h}$ was also performed. Two biologically independent experiments were performed.

parameters, increased serum lactate dehydrogenase (LDH) was significantly associated with poor overall survival and progression-free survival (PFS) rates (22). PPP3CA expression was significantly higher in patients with increased serum LDH levels than those with normal LDH (Figure 1C). These correlations could support the contention that high PPP3CA expression levels might be involved in the pathogenesis of MM. An important role in cell adhesion-mediated drug resistance (CAM-DR) to bortezomib and conventional chemotherapy in MM is played by $\alpha_{4}$ integrins (23). Expression of $\alpha_{4}$ integrins tended to be high in MM patients with elevated PPP $3 C A$ expression, and the possible relevance of high PPP3CA expression to drug resistance was also supposed (Figure 1D).

Panobinostat induces protein degradation of PPP3CA through HSP90 inhibition. Next, we investigated the effects of panobinostat on PPP3CA protein expression in MM cell lines and found it was reduced in pano- 
$\mathbf{A}_{1}$

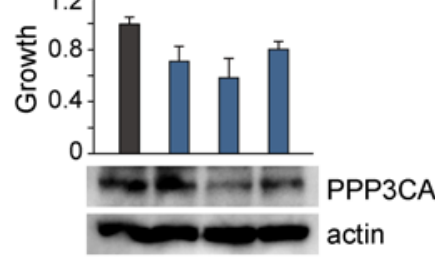

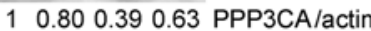

$$
{ }_{5} \text { co }^{x} \frac{x^{2}}{\text { sh-PPP } 3 C A}
$$

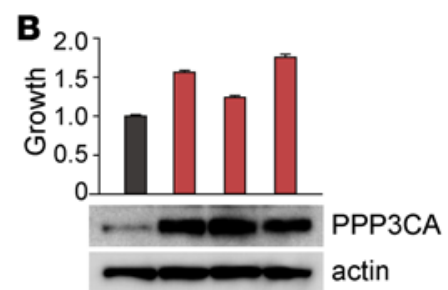

$\begin{array}{lllll}1 & 3.1 & 3.6 & 3.1 & \text { PPP3CA/actin }\end{array}$

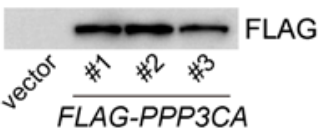

C

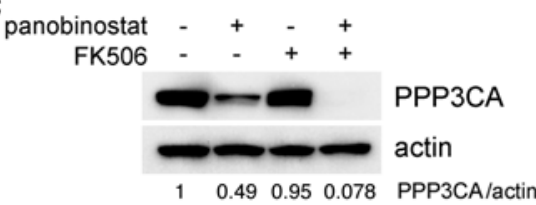

D

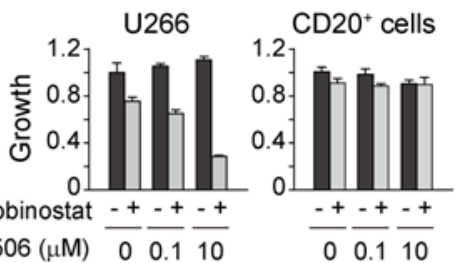

E

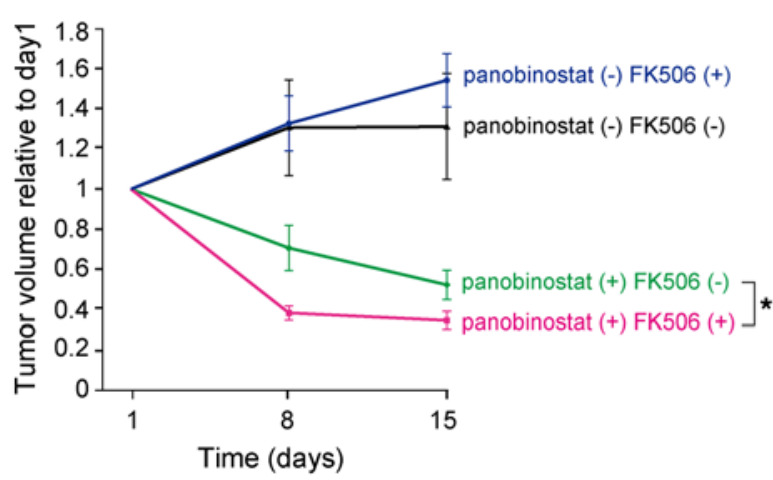

$\mathbf{F}$
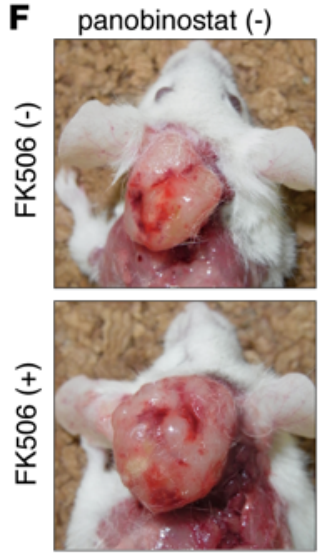

panobinostat $(+)$
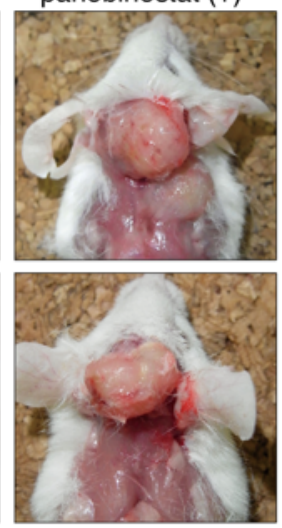

G

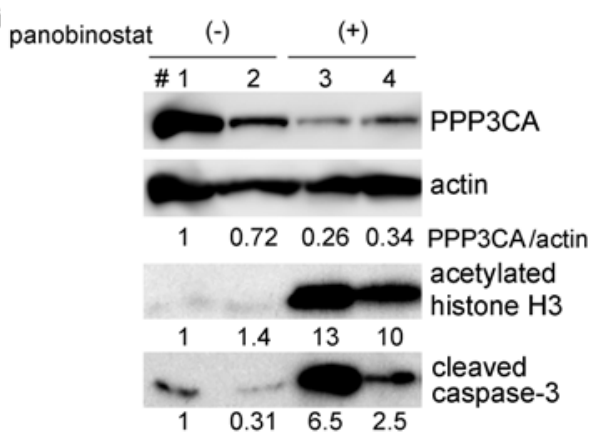

H

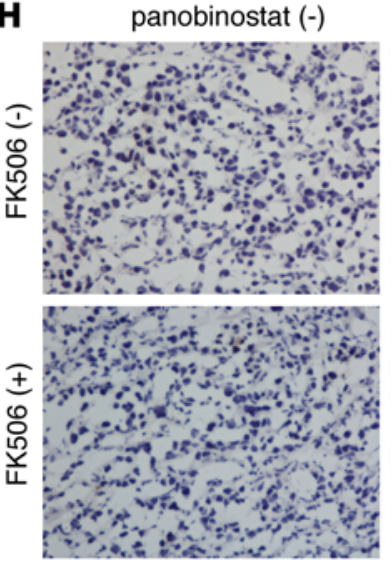

panobinostat $(+)$

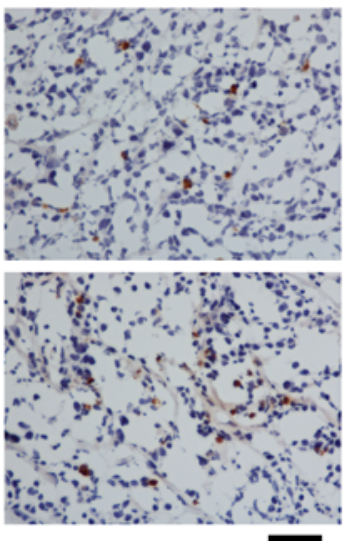

Figure 3. Calcineurin is indispensable for the maintenance of MM cell growth. The degree of protein expression change estimated by quantitative analyses of bands is displayed as indicated. (A) U266 was lentivirally transduced with control vector (sh-cont) or 3 different shRNAs against PPP3CA (KD \#1, \#2, and \#3). Cell growth was determined by MTT assays $(n=5)$, and PPP3CA expression levels are displayed. Two biologically independent experiments were performed. (B) KMS-11 was lentivirally transduced with control vector or FLAG-PPP3CA (clone \#1, \#2, and \#3). Cell growth ( $n=5$ ), and PPP3CA expression are displayed. Four (cell growth) and 2 (PPP3CA expression) biologically independent experiments were performed. (C) U266 was treated with $20 \mathrm{nM}$ panobinostat, $10 \mu \mathrm{M}$ FK506, or both panobinostat and FK506 as indicated for $36 \mathrm{~h}$. Five biologically independent experiments were performed. (D) MTT assays in U266 and CD20-positive cells treated with $15 \mathrm{nM}$ panobinostat, FK506, or both panobinostat and FK506 as indicated for $36 \mathrm{~h}(n=5)$. Three biologically independent experiments were performed. (E) NOD/SCID (NOD/Shilic-scid Jcl) mice bearing U266 cells were treated with vehicle ( $n=6$ ), panobinostat $(n=9)$, FK506 $(n=6)$, or both panobinostat and FK506 $(n=11)$. Treatment was continued for 15 days. Average of the ratio of the tumor volume on days 8 and 15 to that on day 1 is displayed for each condition. Error bars represent the standard errors. Difference between panobinostat (+)/FK506 (-) and panobinostat (+)/FK506 (+) on day 15 was analyzed using Scheffe test. *, significant. (F) The representative images of tumors of each group. (G) Protein expression in tumors of xenograft of mouse model. \#1 and \#2: vehicle treated. \#3 and \#4: panobinostat treated. Two biologically independent experiments were performed. $(\mathbf{H})$ The representative immunohistochemical stainings by anti-cleaved caspase-3 are displayed ( $\times 40)$. The brown cells are positive for staining. Scale bar: 50 microns. Six biologically independent experiments were performed. 
binostat-treated cells compared with mock-treated cells (Figure 2A and Supplemental Figure 2A). However, there was no reduction in PPP3CA mRNA expression (Figure 2A). Calcineurin is a client protein of HSP90, and PPP3CA expression was reduced by the HSP90 inhibitor 17-AAG (Figure 2B and refs. 24, 25). We confirmed the interaction between HSP90 and PPP3CA (Figure 2C). Lactacystin, a proteasome inhibitor, rescued the reduction of PPP3CA induced by panobinostat, thereby supporting the possibility that PPP3CA expression was reduced through protein degradation by panobinostat (Figure 2D).

Inhibition of HDAC6, which primarily deacetylates HSP90, leads to the hyperacetylation of HSP90 and the subsequent disruption of its chaperone function $(15,26,27)$. Treatment with ACY-1215, a selective HDAC6 inhibitor (8), resulted in a stronger reduction in PPP3CA levels than did treatment with panobinostat or romidepsin - an inhibitor of HDAC1, -2, and -3 - though each agent alone induced similar levels of histone $\mathrm{H} 3$ acetylation (Figure 2E). It was shown that PPP3CA reduction by romidepsin occurred mainly through its transcriptional repression (Supplemental Figure 2B). We found that ACY-1215 treatment reduced the association between PPP3CA and HSP90 (Figure 2F). These results suggest that the inhibition of HDAC6 induces PPP3CA degradation by disrupting HSP90 chaperone activity. Lactacystin rescued the reduction of PPP3CA induced by ACY-1215 (Figure 2G).

We showed that part of the antimyeloma effect achieved by HDAC inhibitors is mediated by the inhibition of HSP90. Indeed, HSP90 inhibition, in combination with bortezomib, represents a promising strategy for treating MM (28). However, HSP90 inhibitors, including 17-AAG, are known to activate the heat shock transcription factor 1 (HSF1). HSF1-dependent transcriptional induction of HSP70 protects cancer cells from apoptosis (29). This inhibition of apoptosis is thought to reduce the efficacy of the antitumor effects conferred by HSP90 inhibitors. We confirmed that 17-AAG enhanced protein expression of HSP70 (Figure 2H). In contrast, only a small increase was seen in the expression of HSP70 in samples treated with HADC inhibitors alone or in combination with FK506 (Figure 2I). These results support the idea that calcineurin-targeting therapy might be more accurately compared with conventional HSP90 inhibitors, which can induce HSP70 and thereby dampen any antitumor effects.

Inhibition of calcineurin reduces the growth of MM cells in vitro and in vivo. To evaluate the role of PPP3CA in MM cell viability, we knocked down PPP3CA in MM cells and found that their growth was suppressed in a PPP3CA expression level-dependent manner (Figure 3A). In contrast, overexpression of PPP3CA enhanced the growth of MM cells (Figure 3B), thereby suggesting that PPP3CA expression levels might regulate MM cell growth. The calcineurin complex is composed of PPP3CA and calcineurin $\mathrm{B}$, the regulatory subunit of calcineurin (10). FK506 inhibits the association between PPP3CA and calcineurin B $(30,31)$. To investigate the role of calcineurin B in PPP3CA expression, we examined PPP3CA protein expression in MM cells simultaneously treated with HDAC inhibitors and FK506. PPP3CA expression was more strongly reduced by a combination of panobinostat or ACY-1215 with FK506 compared with HDAC inhibitor alone (Figure 3C and Supplemental Figure 3A). FK506 alone had no suppressive effect on the growth of MM cells. By contrast, FK506 — when used in tandem with panobinostat or ACY-1215 induced a further reduction in cell growth compared with an HDAC inhibitor alone (Figure 3D and Supplemental Figure 3B). $\mathrm{t}(4 ; 14)$ is one of the major chromosomal abnormalities linked to poor prognosis (32). We investigated combinatory effect of panobinostat and FK506 on growth of t(4;14)-positive (KMS-11, KMS-18, and KMS-26) and t(4;14)-negative (U266 and KMS-12PE) cells. Growth of myeloma cells were reduced by panobinostat, and this reduction was enhanced by the addition of FK506 in both t(4;14)-positive and -negative myeloma cell lines (Supplemental Figure 3C and refs. 33-35). Of note, panobinostat did not exert an overt effect on cell growth in normal B cells, and FK506 did not augment its cytotoxic effect (Figure 3D). Next, we studied the pathogenic role of calcineurin in MM in vivo using a xenograft mouse model of myeloma cells. Tumor sizes tended to increase in control and FK506-treated mice. In contrast, it decreased in panobinostat-treated mice. This reduction in tumor size was further enhanced by combined treatment with panobinostat and FK506 for 15 or 22 days (Figure 3, E and F, and Supplemental Figure 4, A-C). Reduced PPP3CA expression, enhanced histone H3 acetylation, and cleavage of caspase- 3 in vivo were confirmed in the xenograft samples of panobinostat mice (Figure 3G). Further, the addition of FK506 augmented panobinostat-induced apoptosis in vivo (Figure $3 \mathrm{H}$ ). Taken together, the addition of FK506 enhances the antimyeloma effect of panobinostat through PPP3CA reduction, thereby supporting the importance of calcineurin in the pathogenesis of MM.

The effects of calcineurin inhibition on apoptosis and proliferation of MM cells and the identification of functional targets of PPP3CA in MM. We studied whether induction of apoptosis and/or suppression of MM cell 


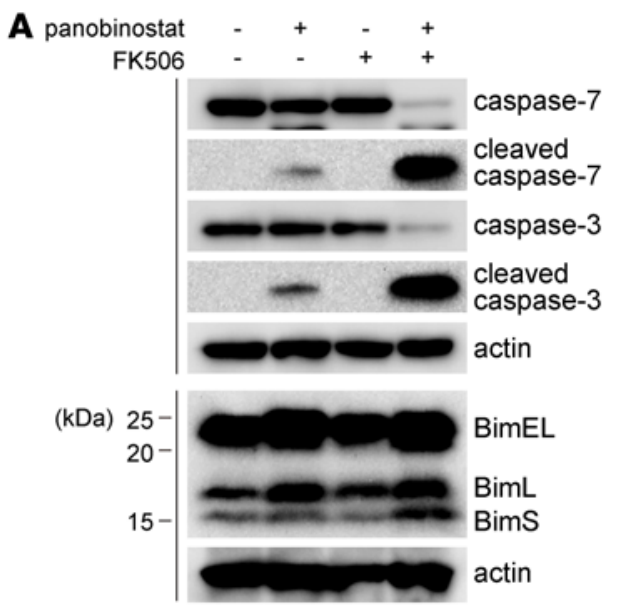

B

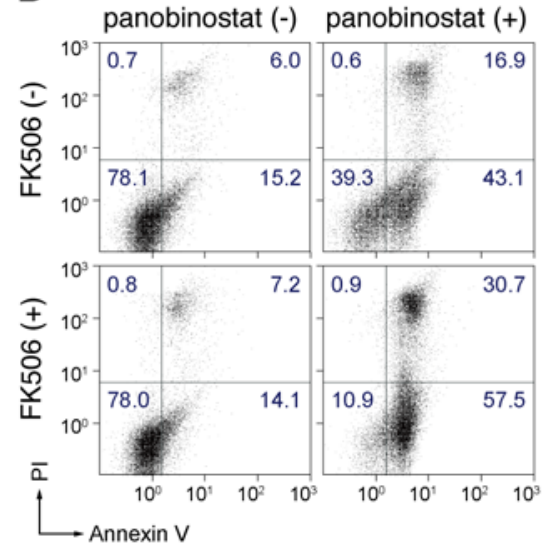

C

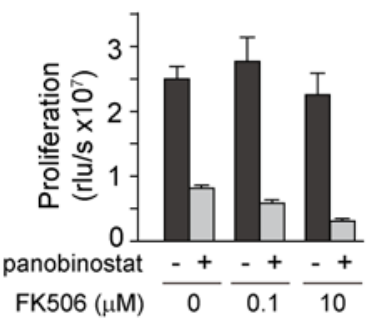

D

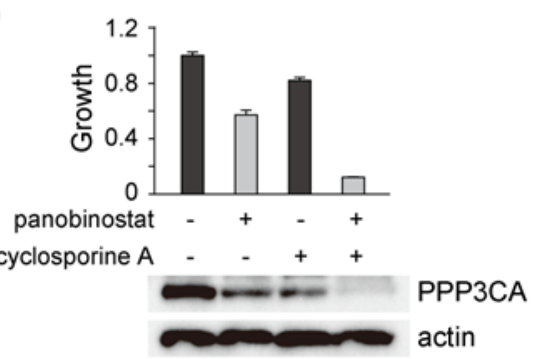

E

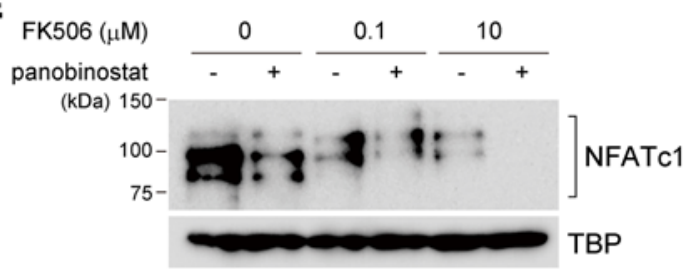

$\mathbf{F}$

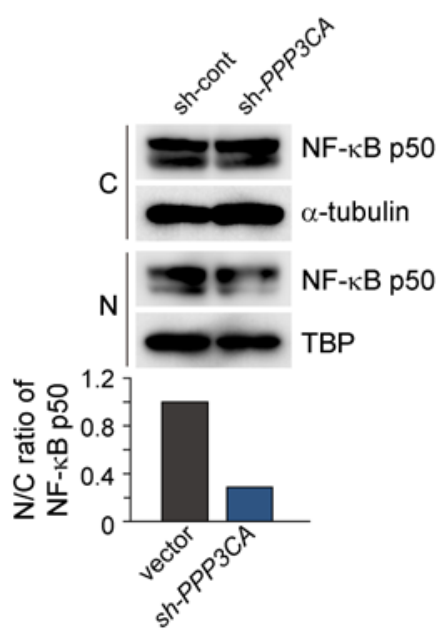

G

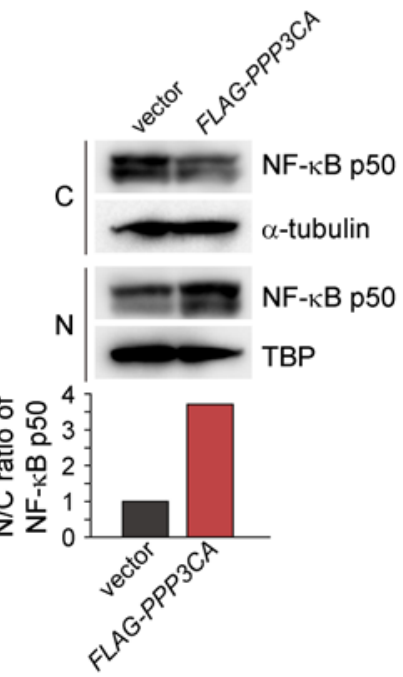

H

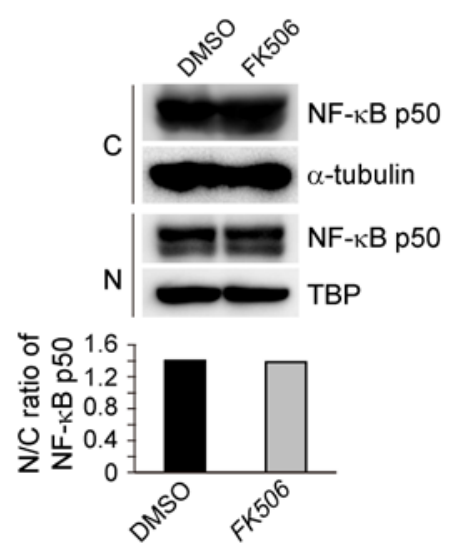

Figure 4. Calcineurin inhibition enhances apoptosis and blocks proliferation, and PPP3CA regulates NF-KB signaling in MM cells. (A) Apoptotic protein expression of KMS-11 treated with $20 \mathrm{nM}$ panobinostat, $10 \mu \mathrm{M}$ FK506, or both panobinostat and FK506 for $36 \mathrm{~h}$ is displayed. Two biologically independent experiments were performed. (B) KMS-11 was treated with $20 \mathrm{nM}$ panobinostat, $10 \mu \mathrm{M}$ FK506, or both panobinostat and FK506 for $36 \mathrm{~h}$. Induced apoptosis was evaluated by flow cytometry. Annexin V (+) and propidium iodide (PI) (-): early apoptotic cells. Annexin V (+) PI (+): late apoptotic cells. Two biologically independent experiments were performed. (C) BrdU assays in KMS-11 treated with $15 \mathrm{nM}$ panobinostat, FK506, or both panobinostat and FK506 as indicated for $48 \mathrm{~h}(n=6)$. Two biologically independent experiments were performed. (D) Cell growth and PPP3CA expression in U266 treated with $20 \mathrm{nM}$ panobinostat, $10 \mu \mathrm{M}$ cyclosporine A, or both panobinostat and cyclosporine A as indicated for $48 \mathrm{~h}(n=5)$. Two biologically independent experiments were performed. (E) NFATc1 (nuclear factor of activated T cells, cytoplasmic, calcineurin-dependent 1) protein in U266 treated with $20 \mathrm{nM}$ panobinostat, FK506, or both panobinostat and FK506 as indicated for $72 \mathrm{~h}$. TATA-binding protein (TBP) served as a loading control. Four biologically independent experiments were performed. (F and G) Cytoplasmic (C) and nuclear (N) NF-KB p50 in U266 lentivirally transduced with control vector (sh-cont) or shRNA against PPP3CA (sh-PPP3CA) (F) and control vector (vector) or PPP3CA (FLAG-PPP3CA) (C). $\alpha$-tubulin and TBP served as a loading control. N/C ratios of NF- $\kappa B$ p50 are also displayed. Two biologically independent experiments were performed. (H) C and N NF-KB p50 in U266 treated with DMSO or $10 \mu \mathrm{M}$ FK506 for $48 \mathrm{~h}$ followed by Western blot analysis. Two technically independent experiments were performed. 

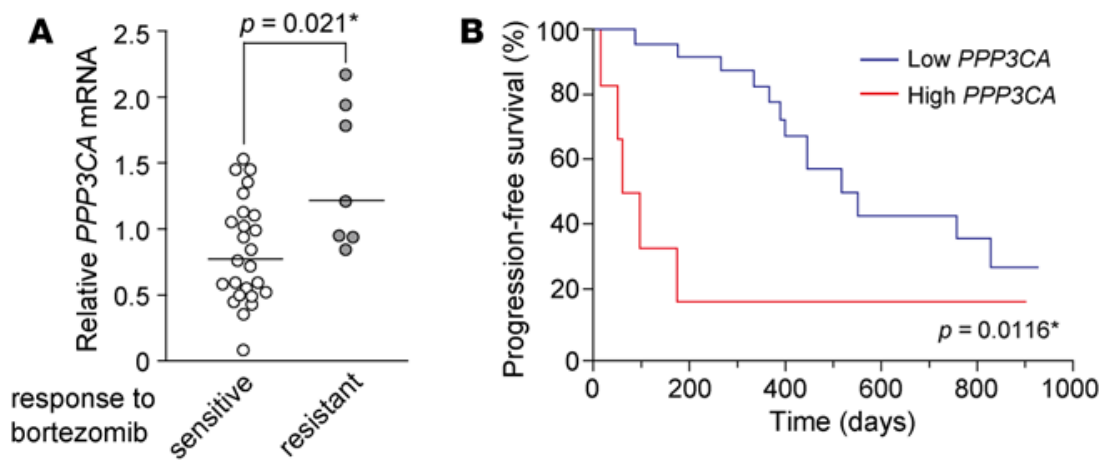

\section{C}
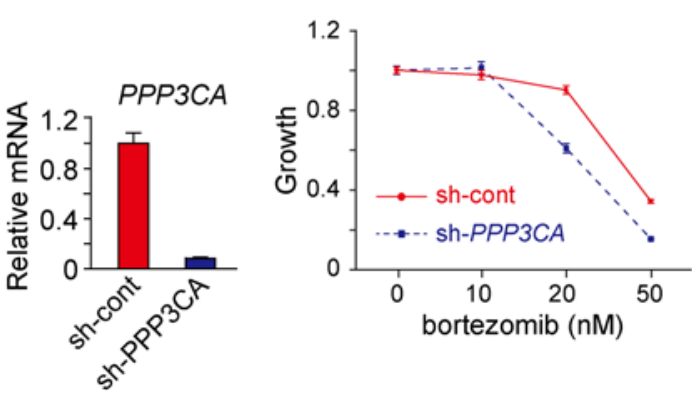

D
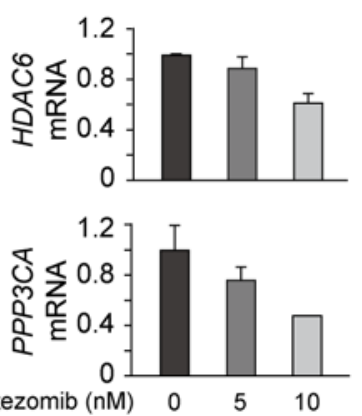

bortezomib $(\mathrm{nM})$

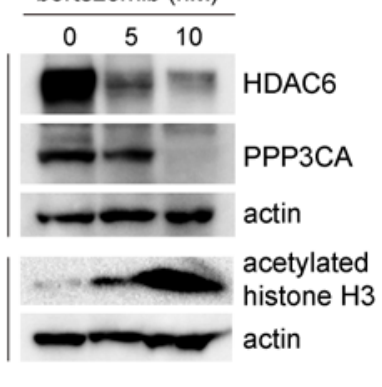

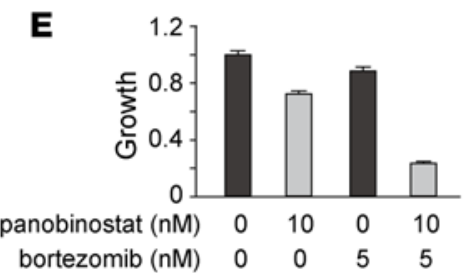

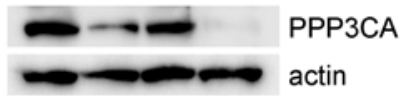

$\mathbf{F}$
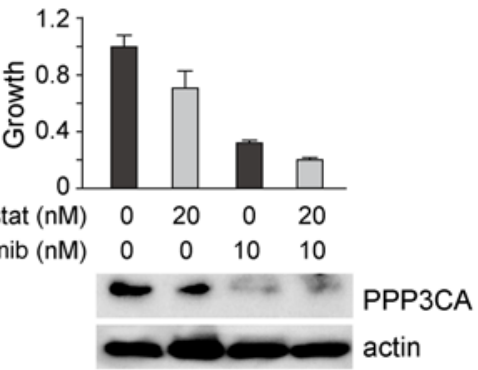

Figure 5. PPP3CA is a common target of panobinostat and bortezomib. (A) PPP3CA mRNA expression in MM patients treated with VMP (bortezomib, melphalan, and prednisone) or BD (bortezomib, dexamethasone). Sensitive: patients with partial response or better $(n=25)$. Resistant: patients with stable disease or death during therapy $(n=7)$. Horizontal lines, median. Difference between 2 groups of samples were analyzed using 1-tailed $t$ test. *, significant. (B) Kaplan-Meier curve of progression-free survival (PFS) of MM patients treated with VMP or BD. Low PPP3CA ( $n=26)$ : relative PPP3CA mRNA is less than 1.5. High PPP3CA $(n=6)$ : relative PPP3CA mRNA is 1.5 or more. The difference in PFS between the 2 groups was analyzed using a Log-rank test. *, significant. (C) U266 transduced with a control vector (sh-cont) or shRNA against PPP3CA (KD \#2) was treated with bortezomib as indicated for $48 \mathrm{~h}$. PPP3CA mRNA expression was estimated by analyses of 6 samples for each clone. The ratio of MTT values at each bortezomib concentration to that at $0 \mathrm{nM}$ is displayed $(n=5)$. Similar results were obtained for KD \#1 and \#3 (data not shown). Two biologically independent experiments were performed. (D) HDAC6 and PPP3CA expression in U266 treated with bortezomib as indicated for $12 \mathrm{~h}$ (mRNA analysis) or $72 \mathrm{~h}$ (protein analysis). Three (mRNA analysis: $n=2)$ and 2 (protein analysis) biologically independent experiments were performed. (E) Cell growth $(n=5)$ and PPP3CA expression levels in U266 treated with panobinostat, bortezomib, or both panobinostat and bortezomib for $72 \mathrm{~h}$. Three (cell growth) and 2 (PPP3CA expression) biologically independent experiments were performed. (F) Cell growth $(n=5)$ and PPP3CA expression in KMS-11 treated with $20 \mathrm{nM}$ panobinostat and $10 \mathrm{nM}$ carfilzomib for $24 \mathrm{~h}$. Two biologically independent experiments were performed.

proliferation would contribute to the anti-MM effects observed in the simultaneous treatment with HDAC inhibitors and FK506. Combined treatment with panobinostat and FK506 enhanced cleavage of caspase-7 and -3 while increasing the BCL2-family protein BimS expression compared with panobinostat alone (Figure 4A). The previous study showed that Bim gene KO mouse embryo fibroblasts displayed diminished HDAC inhibitor-mediated apoptosis (36). These results suggest the importance of enhanced expression of Bim in HDAC inhibitor-mediated apoptosis and the possibility of blocking the effect of combined treatment with panobinostat and FK506 by shRNA Bim knockdown. Flow cytometric analysis confirmed that FK506 enhanced MM apoptosis induced by panobinostat or ACY-1215 (Figure 4B and Supplemental Figure 5). In addition, the proliferation of MM cells was inhibited by panobinostat, an inhibition that was enhanced by the addition of FK506 (Figure 4C). The combination of panobinostat and cyclosporine 
A, another calcineurin B inhibitor, also increased the reduction of cell viability and PPP3CA expression induced by panobinostat (Figure 4D).

The NFATc1 is one of the PPP3CA substrates. The NFATc1 dephosphorylated by PPP3CA translocates from the cytoplasm to the nucleus, and its nuclear translocation is blocked by FK506 (37). We found that combined treatment with panobinostat and FK506 not only substantially suppressed nuclear NFATc1 expression, but also blocked calcineurin signaling (Figure 4E). FK506 alone suppressed nuclear NFATc1 expression. However, the viability of MM cells was not affected by FK506. Moreover, dephosphorylation of NFATc1 by PPP3CA does not appear to be related to those signals that promote MM survival (Figure 3D).

In addition to mediating NFATc1 activation, PPP3CA was shown to synergize with protein kinase $\mathrm{C}(\mathrm{PKC})$ in order to activate NF- $\mathrm{KB}$ signaling in $\mathrm{T}$ cells (38). The latter is involved in the pathogenesis of $\mathrm{MM}$ (39). Panobinostat disrupts formation of the NF- $\mathrm{kB} / \mathrm{DNA}$ binding complex, thereby inactivating NF- $\mathrm{KB}$ signaling (40). This inactivation may be due to downregulation of PPP3CA by panobinostat. In fact, PPP3CA knockdown reduced the nuclear/cytoplasmic ratio of NF- $\mathrm{KB}$ p50 subunit expression in MM cells (Figure 4F). Furthermore, its overexpression increased that ratio (Figure 4G). These results suggest that PPP3CA plays an important role in NF- $\mathrm{kB}$ signal activation in MM cells. Thus, it is possible that panobinostat reduces MM cell viability by suppressing PPP3CA-NF-кB signaling. On the other hand, FK506

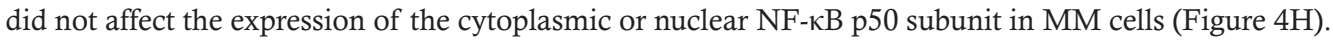

PPP3CA is a common target of panobinostat and bortezomib. Next, we examined whether the PPP3CA reduction induced by panobinostat is relevant to the clinical effect that panobinostat adds to bortezomib therapy $(1,2)$. We examined a correlation between PPP3CA expression in CD138-positive cells and the clinical responses to bortezomib-containing therapies in newly diagnosed patients treated with VMP (bortezomib, melphalan, and prednisone regimen) or $\mathrm{BD}$ (bortezomib plus dexamethasone therapy) at 2 centers from 2012-2014 (Supplemental Table 1). PPP3CA expression was significantly higher in bortezomib-resistant patients versus those who were bortezomib sensitive (Figure 5A). Furthermore, PFS duration in patients with high $P P P 3 C A$ expression was shorter than that of patients with low $P P P 3 C A$ expression in bortezomib-containing therapy (Figure 5B). PPP3CA knockdown by lentiviral shRNA (Figure 5C, left) enhanced the sensitivity to bortezomib compared with controls (Figure $5 \mathrm{C}$, right). Thus, $P P P 3 C A$ expression levels correlated with bortezomib resistance. Bortezomib suppressed not only mRNA and protein expression of HDAC6 in a dose-dependent manner, but also PPP3CA protein expression (Figure 5D). Induction of protein acetylation by bortezomib was confirmed by hyperacetylation of histone $\mathrm{H} 3$ in MM cells treated with bortezomib (Figure 5D). These results indicate that bortezomib may suppress PPP3CA through HDAC6 inhibition. In fact, $P P P 3 C A$ was transcriptionally suppressed by bortezomib (Figure 5D). Furthermore, cotreatment with panobinostat and bortezomib additively suppressed cell viability (Figure $5 \mathrm{E}$ ). These results suggest that panobinostat might augment the antimyeloma effect of bortezomib by further reducing PPP3CA expression. Additive reduction of MM cell viability and PPP3CA expression was also observed in another combination treatment paring panobinostat with carfilzomib, another proteasome inhibitor (ref. 41 and Figure 5F). Suppression of cell viability and PPP3CA were enhanced by cotreatment with ACY-1215 and bortezomib (Supplemental Figure 6A). The antimyeloma effect of bortezomib was enhanced by cotreatment with FK506 (Supplemental Figure 6B). Combinatory treatment with panobinostat and bortezomib reduced cell growth both in vector and FLAG-PPP3CA-introduced cells compared with vehicle treatment. However, the growth ratio of PPP3CA overexpressed cells to that of control cells was comparable between vehicle and drug-treated cells. Thus, combination strategy did not block the growth enhancement induced by ectopic overexpression of PPP3CA (Supplemental Figure 7).

Formation of osteoclasts is inhibited by panobinostat. MM generates lytic bone lesions via the formation of osteoclasts (42). Osteoclast differentiation requires the induction of NFATc1 by receptor activator nuclear factor- $\kappa-B$ ligand (RANKL). As FK506 inhibits osteoclast differentiation (43), we investigated the effect of panobinostat treatment on the latter. Panobinostat and FK506 each inhibited osteoclast differentiation, while the addition of both drugs strengthened the blockade of osteoclast formation compared with treatment with either agent alone (Figure 6A). PPP3CA protein levels, but not mRNA expression, decreased in differentiated osteoclasts treated with panobinostat (Figure 6B). NFATc1 activation is required for the induction of essential osteoclast transcription factors, such as microphthalmia-associated transcription factor (MITF) (44). In addition to the NFATc1-MITF pathway, MITF is upregulated by HSP70 (44). Although 17-AAG is a known potent cytotoxic drug, it is associated with side effects that cause bone loss due to increases in HSP70-MITF. In osteoclasts treated with panobinostat, MITF was reduced without any 
A

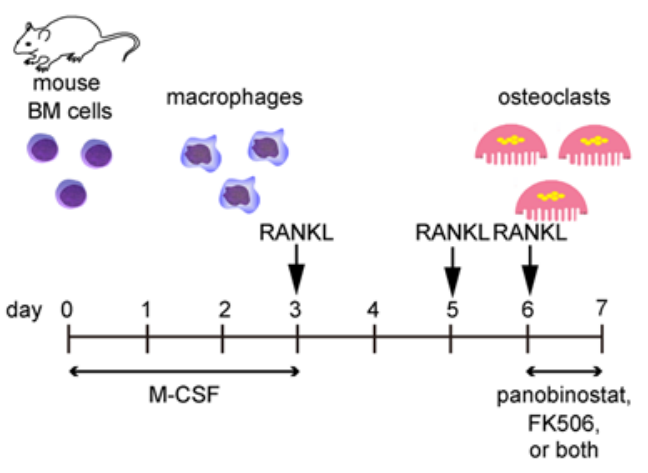

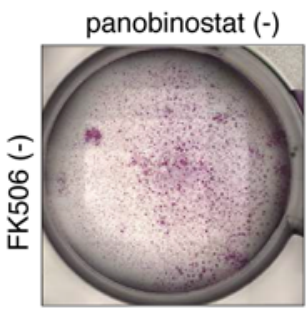
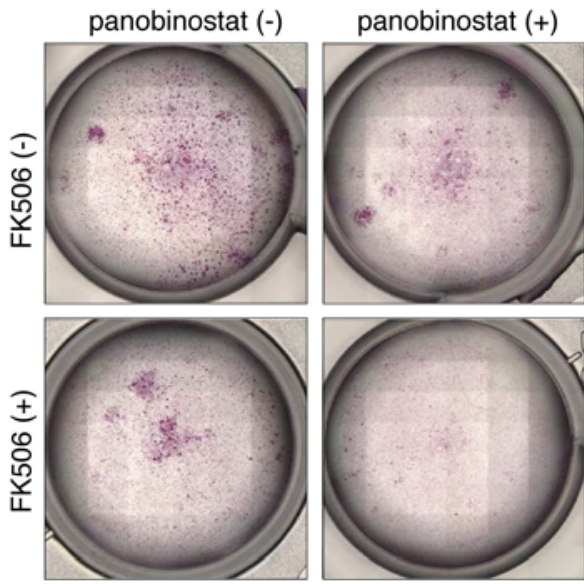

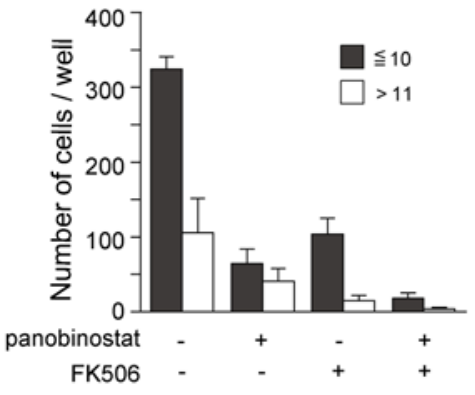

B

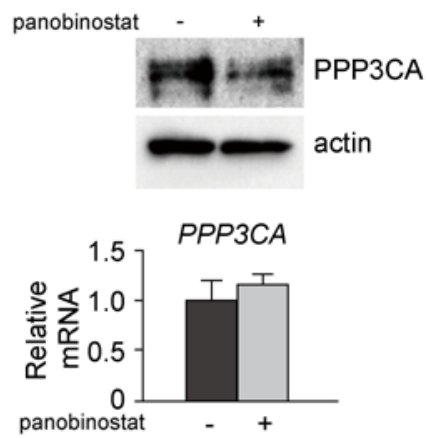

C panobinostat

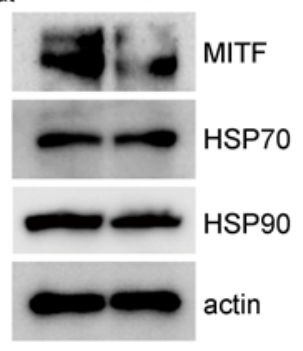

Figure 6. Panobinostat blocks osteoclast formation. (A) Panobinostat ( $5 \mathrm{nM}$ ), FK506 (100 nM), or both panobinostat and FK506 were added to the medium after the third stimulation of macrophages differentiated from mouse BM mononuclear cells by RANKL (receptor activator nuclear factor- $\kappa$ - $B$ ligand). The cells were cultured for $24 \mathrm{~h}$ as illustrated. M-CSF, macrophage colony-stimulating factor. Images and colony counts of the differentiated osteoclasts in each condition are displayed $(n=4)$. Black, colonies with 10 or fewer nuclei. White, colonies with 11 or more nuclei. Three biologically independent experiments were performed. (B) PPP3CA protein and PPP3CA mRNA expression $(n=3)$ in osteoclasts treated with $1 \mathrm{nM}$ panobinostat for $24 \mathrm{~h}$ after stimulation by RANKL. Two biologically independent experiments were performed. (C) Protein expression in osteoclasts treated with $1 \mathrm{nM}$ panobinostat for $24 \mathrm{~h}$ after stimulation by RANKL. Two biologically independent experiments were performed.

increase in HSP70 (Figure 6C). These results suggest that treatment of MM with panobinostat would not cause osteoclast activation, as seen in 17-AAG treatment.

\section{Discussion}

In this study, we have shown the possible pathogenesis of PPP3CA, a catalytic subunit of calcineurin, in MM. In 4q22.3-4q24, where PPP3CA is located, gains in both copy number and gene amplification have been reported based on analyses of MM patient tumors (19). This suggests that high PPP3CA expression plays a pathogenic role in MM. Analyses of data from previous reports, and from our clinical samples, revealed that PPP3CA expression was higher in the tumors of advanced MM patients (stage III) than in those of nonadvanced patients (stage I). In addition, increased serum LDH in patients with high $P P P 3 C A$ expression points to its potential relevance in poor prognosis. Furthermore, it has been suggested that PPP3CA maintains MM cell viability through activation of NF- $\mathrm{kB}$ signaling. Cotreatment of MM cells with panobinostat and FK506 synergistically reduced PPP3CA and MM cell viability. On the other hand, the cytotoxic effect exerted by panobinostat on $\mathrm{CD} 20^{+}$cells was subtle, while the addition of FK506 did not increase their viability. Moreover, development of $\mathrm{T}$ and $\mathrm{B}$ lineage cells was normal in PPP3CA-deficient mice, and panobinostat did not compromise donor lymphocyte reconstitution in a mouse BM transplantation model $(45,46)$. These results suggest that calcineurin-targeting therapy exerts an antimyeloma effect without inducing significant side effects in normal lymphoid systems. The 5TGM1/Kalwrij model of myeloma is an immune-competent model involving bone lesions and is considered suitable for analyses of the in vivo effects of antimyeloma drugs on $\mathrm{T}$ cell populations, as well as osteoclasts activity (47). However, treatment with FK506 significantly suppressed cellular immunity in immune competent mice (48). Transfer 
B cells deficient in Blimp1 (the tumor suppressor gene) into mice lacking T cells ( $\left.\operatorname{Rag}^{{ }^{-/}}\right)$resulted in mutant cells expansion, but this transfer into WT mice did not in the previous study (49). Thus, it is supposed that $\mathrm{B}$ cell malignant cells will expand more in T cell-suppressed mice than control mice. These results suggest the possibility of enhanced growth of transplanted 5TGM1 cells by FK506 in a 5TGM1/Kalwrij model of myeloma. On the other hand, in our xenograft immunocompromised mouse model, tumor volume was comparable between panobinostat (-)/FK506 (-)-treated and panobinostat (-)/FK506(+)-treated mice (Figure 3E and Supplemental Figure 4A). Furthermore, myeloma cells are responsible for producing osteoclastogenic activity (50), and FK506 is supposed to modify the osteoclast activity through enhanced growth of myeloma cells in the 5TGM1/Kalwrij model of myeloma. For this reason, the 5TGM1/Kalwrij model of myeloma may not be appropriate for analyzing the effects of combined HDAC inhibitors and FK506 on implanted tumors and osteoclasts activity.

Recently, the activity of HDACs vis-à-vis nonhistone proteins such as HSP90 has attracted increasing attention as a key aspect of HDAC function (15). We discovered that HDAC inhibitors disturbed PPP3CA through inhibition of HSP90. ACY-1215 is a specific inhibitor of HDAC6, a class IIb HDAC (15). HDAC6 maintains HSP90 activation via its acetylation and prevents degradation of its client proteins. HDAC6-specific inhibition is not associated with disrupted epigenetic control of gene transcription, as has been observed in the usage of pan-HDAC inhibitors such as panobinostat (51). We investigated the effect of ACY-1215 on PPP3CA in order to elucidate the role HDAC6 plays in the protein stability of PPP3CA. Treatment of MM cells by ACY-1215 degraded PPP3CA with a reduction in cell viability. This degradation was mediated by the release of PPP3CA from HSP90. HDAC6-mediated acetylation of HSP90 might play an important role in the maintenance of PPP3CA stability and MM cell viability. HDAC1-3 downregulate their targets through transcriptional repression. Epigenetic recovery of tumor-suppressor genes such as p21 has been linked to the actions of HDAC inhibitors, including panobinostat $(15,52)$. However, some oncogenes such as CCND2 and Ki-67 are known to be suppressed by HDAC inhibitors (53). The reduction of $P P P 3 C A$ mRNA by romidepsin suggested that PPP3CA could be the target of both protein degradation and transcriptional repression by HDAC inhibitors.

Although FK506 by itself neither affects MM cell viability nor reduces PPP3CA expression, its use in cotreatment with HDAC inhibitors negatively impacts both. Calcineurin B has been shown to protect PPP3CA from protein degradation in in vitro experiments (54). This indicates that the ability of FK506 to additively inhibit any interaction between PPP3CA and calcineurin B promotes the degradation of unstable PPP3CA released from HSP90 by HDAC inhibitors. Thus, FK506 is supposed to enhance the antimyeloma effects of HDAC inhibitors. High expression of $P P P 3 C A$ will be important since PPP3CA mediates myeloma cell growth through PPP3CA-NF- $\mathrm{KB}$ signaling and could be the target of those HDAC inhibitors supported by FK506. The authors believe that this cotreatment would be clinically beneficial for a certain group of MM patients, as will be discussed in detail herein. Reduced intensity-conditioned allogeneic stem cell transplantation (RIST) is a tolerable and effective treatment for some MM patients who relapse after autologous stem cell transplantation (55). However, the reduced intensity of conditioning therapy may lead to the presence of residual disease. Although immunosuppressive drugs (e.g., FK506) are used to facilitate allograft survival and prevent graft-versus-host disease, they increase the risk of expanded residual disease and relapse. The enhanced antimyeloma effect of combination therapy (panobinostat + immunosuppressive drugs) may aid panobinostat-maintenance therapy in those patients receiving RIST. Approval of salvage therapy by panobinostat for relapsed and refractory patients indicates the possible effectiveness of such maintenance therapy. The results of our xenograft mouse model experiments have shown FK506's additive effect on the antimyeloma effect exerted by panobinostat. In these experiments, panobinostat and FK506 were safely administered in vivo without a significant loss in body weight. The minimum dosage for oral administration of FK506 necessary to suppress immunity in vivo was $10 \mathrm{mg} / \mathrm{kg}$ body weight for 4 days (total $40 \mathrm{mg} / \mathrm{kg}$ for a week) in a previous study (48). In a clinical setting, a noncontinuous dosing schedule of panobinostat (e.g., 3 times per week) is necessary to reduce the risk of thrombocytopenia (52). To enhance these antimyeloma effects, concurrent administration of panobinostat and FK506 should prove an effective therapy. Thus, $10 \mathrm{mg} / \mathrm{kg}$ of oral administration of FK506 (total $30 \mathrm{mg} / \mathrm{kg}$ for a week) is believed to be an appropriate and efficient treatment. Tumor volume differences between panobinostat $(+) / F K 506$ $(-)$-treated and panobinostat $(+)$ /FK506 (+)-treated mice were significant, as determined by Scheffe test analyses, both on days 8 and 15 (treatment for 15 days) and on days 8,15 , and 22 (treatment for 22 days) (Supplemental Figure 4, B and C). The ratio of tumor volume in panobinostat (+)/FK506 (+)-treated mice 


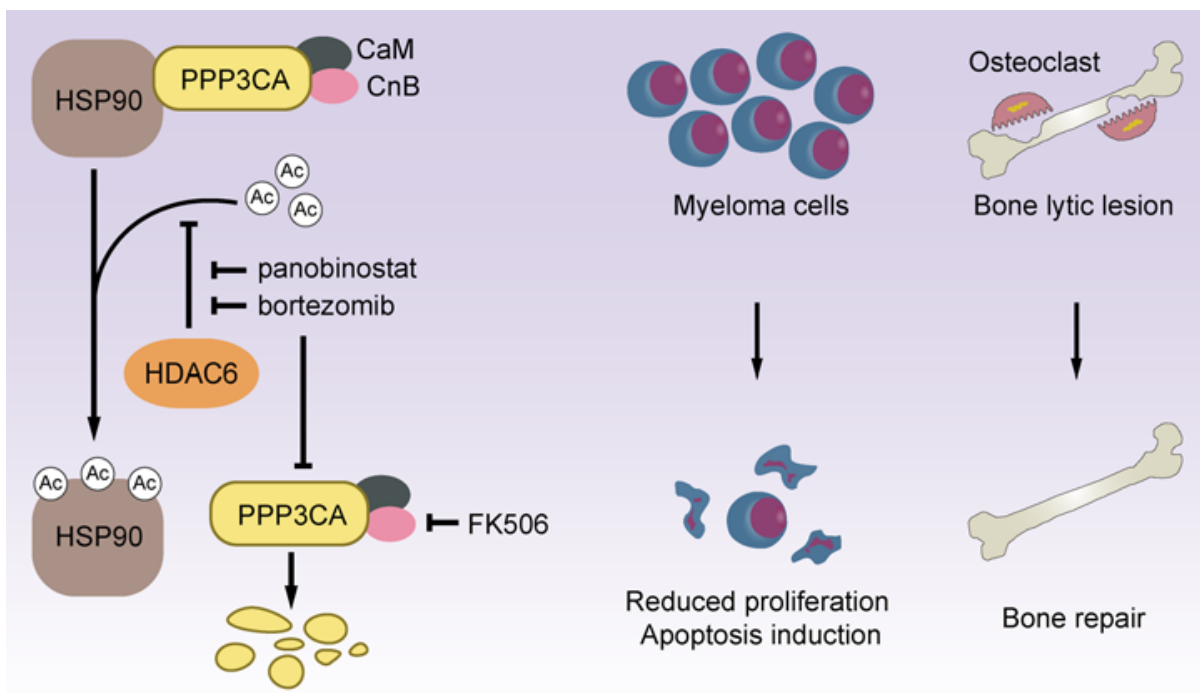

Figure 7. Proposed molecular mechanism of the clinical effects of calcineurin-targeting therapy in MM patients. Schematic representation of the reduction in PPP3CA induced by panobinostat and bortezomib and the expected clinical effects of PPP3CA reduction in MM patients are displayed. CaM, calmodulin; CnB, calcineurin B; Ac, acetylation.

versus panobinostat (+)/FK506 (-)-treated mice decreased on day 22 (0.459) compared with that on day 15 (0.623) (Supplemental Figure 4C). These results indicate that combinatory therapy for 22 days is more effective than that for 15 days. Thus, we believe that treatment should be carried out for at least 22 days.

Bortezomib plays a central role as a drug in MM therapies, both in transplantation-eligible and -ineligible patients. However, many patients develop a resistance to bortezomib during therapy, thereby impeding their long-term survival. Such resistance must be overcome if MM patients are to enjoy improved survival rates. Panobinostat, an FDA-approved drug, when used in combination with bortezomib is expected to overcome this resistance. Proteasome inhibition by bortezomib leads to the formation of aggresomes, which are dependent upon the interaction of HDAC6 with tubulin and the dynein complex (5). HDAC6 inhibition induces hyperacetylation of tubulin and blocks aggresome formation, which is accompanied by increased cellular stress and apoptosis (3). These results indicate that panobinostat enhances the antimyeloma effect of bortezomib by inhibiting aggresome formation. We found that high PPP $3 C A$ expression is indicative of a poor prognosis due to resistance to bortezomib. Thus, we believe that PPP3CA could serve as a target of therapy in bortezomib-resistant patients. In addition, we examined the expression of $P P P 3 C A$ and several genes associated with bortezomib resistance (PSMB5, CDK5, and CYP1A1) in patients treated with bortezomib as well as dexamethasone in the millennium trial (56-59). We compared the expression of each gene between responsive $(\mathrm{R})$ (minor response and better) and nonresponsive (NR) (no change and progressive disease) patients. There was no significant difference of expression in CDK5 and CYP1A1 between $\mathrm{R}$ and NR. However, $P S M B 5$ expression was substantially higher in NR compared with $\mathrm{R}$, and a trend of higher expression of $P P P 3 C A$ in NR compared with $\mathrm{R}$ was evident (Supplemental Table 2). The relevance of high $P P P 3 C A$ expression vis-à-vis bortezomib resistance should be investigated by further analysis in a large cohort treated with a unified bortezomib-containing protocol. We have shown that bortezomib and panobinostat synergistically reduce MM cell viability through PPP3CA reduction. The antimyeloma effects of this combined therapy mediated inhibited aggresome formation and synergistic PPP3CA reduction. This is consistent with the clinical findings about the additive effect panobinostat provides to bortezomib and dexamethasone therapy $(1,2) . \alpha_{4}$ integrins are associated with CAM-DR, and the correlation between $P P P 3 C A$ and $\alpha_{4}$ integrins expression in CD138-positive cells from MM patients was found. The reason for poor responses to VMP or BD treatments and short PFS durations in patients with high $P P P 3 C A$ expression may be explained by the pathogenicity of high $P P P 3 C A$ expression, as well as CAM-DR, resulting from high $\alpha_{4}$ integrin expression.

Bortezomib has been shown to not only exert a tumoricidal activity, but also to improve lytic bone lesions in MM patients. Moreover, it has been shown to suppress the formation of osteoclasts by blocking the NFATc1 induction caused by tumor necrosis factor receptor-associated factor 6 (TRAF6) (60). NFATc1 
induction by calcineurin signaling also plays an important role in osteoclast formation (43). In this study, we have demonstrated that panobinostat disturbed osteoclast formation and suppressed PPP3CA. This finding is in accordance with a previous study in which panobinostat reduced bone density loss in a disseminated MM xenograft mouse model (61). Furthermore, addition of FK506 to panobinostat enhanced the blocking of osteoclast formation compared with treatments with either agent alone. This finding indicated that calcineurin-targeted therapy, using a combination of panobinostat and FK506, effectively inhibits lytic bone lesions, as well as MM cell viability. This might serve as a useful tool for halting the cycle induced by MM cells and osteoclasts.

In summary, we have revealed PPP3CA, a catalytic subunit of calcineurin, as a molecule related to both MM cell viability and osteoclast formation (Figure 7). PPP3CA is protected from protein degradation by binding to HSP90, and its degradation was induced by panobinostat and bortezomib through HDAC6 inhibition. Addition of FK506 enhanced PPP3CA reduction induced by panobinostat, and bortezomib has also transcriptionally suppressed $P P P 3 C A$. Furthermore, PPP3CA was related to bortezomib resistance. The development of new calcineurin-targeted therapies, which inhibit PPP3CA-NF- $\mathrm{B}$ signaling by small molecules, is expected to profoundly improve the treatment of MM. This will overcome drug resistance and improve osteolytic lesions in a wide range of patients, including those receiving RIST who may be treated with panobinostat and FK506.

\section{Methods}

Gene expression analysis of candidate oncogenes in MM patients. mRNA expression data on candidate oncogenes from MM patient samples obtained during a previous study (20) were analyzed using the GEO (http:// www.ncbi.nlm.nih.gov/geoprofiles/11574557). Statistical analyses examining the difference of expression of PSMB5, PPP3CA, CDK5, and CYP1A1 between R (complete response [ $n=14]$, partial response $[n=$ $76]$, and minor response $[n=23]$ ) and NR (no change $[n=60]$ and progressive disease $[n=66]$ ) patients who were treated with bortezomib $(n=169)$ or dexamethasone $(n=70)$ in millennium trial (59) were performed using GEO2R (National Center for Biotechnology Information, http://www.ncbi.nlm.nih.gov/ geo/query/acc.cgi?acc=GSE9782, GPL96 platform).

Patient samples. CD138-positive cells were sorted from BM samples (Tokyo Women's Medical University and Nippon Medical School). The patient characteristics are described in Supplemental Table 1. Sensitivity to bortezomib-containing therapies was estimated in those patients who were treated with VMP (bortezomib, melphalan, and prednisone) or BD (bortezomib and dexamethasone).

Cell lines. Human MM (U266, KMS-11, KMS-18, KMS-12PE, KMS-26, and RPMI8226) and nonMM (HEL, HL60, KG-1, THP-1, U937, BALL-1, NALM6, Jurkat, Molt-4, K562, Daudi, and Raji) cell lines were cultured in RPMI-1640 medium supplemented with $10 \%$ FCS, penicillin, and streptomycin. HEK293T cells were cultured in DMEM supplemented with $10 \%$ FCS, penicillin, and streptomycin. The source of cell lines is as follows: U266, RPMI8226, HEL, HL60, KG-1, THP-1, U937, Jurkat, Molt-4, K562, Daudi, Raji, and HEK293T (ATCC); KMS-11 and NALM6 (JCRB cell bank, National Institutes of Biomedical Innovation, Health and Nutrition); KMS-18 and KMS-26 (T. Otsuki, Kawasaki Medical School, Okayama, Japan); and BALL-1 (Riken BioResource Center).

Reagents. Panobinostat(LBH589)(Selleck Chemicals), romidepsin (Selleck Chemicals), ACY-1215 (Chemietek), bortezomib (Selleck Chemicals), FK506 monohydrate (Sigma-Aldrich), cyclosporine A (SigmaAldrich), 17-(allyamino)-17-demethoxygeldanamycin (17-AAG) (Sigma-Aldrich), lactacystin (Santa Cruz Biotechnology Inc.), and carfilzomib (Selleck Chemicals) were dissolved in DMSO and added to the culture medium at the indicated concentrations. Panobinostat for in vivo study was kindly provided by Novartis Pharma Inc. (Fabrikstrasse 28-Z1.28.1 Novartis Campus CH-4056). FK506 for in vivo study was provided by Astellas Pharma Inc.

Western blot analyses and immunoprecipitation. Total cell lysates were prepared as follows. Collected cells were washed with PBS and lysed in RIPA buffer (50 mM Tris- $\mathrm{HCl}$ [pH 7.4], $150 \mathrm{mM} \mathrm{NaCl}, 1 \% \mathrm{NP}-40$, $0.1 \%$ SDS, $0.1 \%$ deoxycholate, $1 \mathrm{mM}$ phenylmethylsulfonyl fluoride [PMSF], $2 \mu \mathrm{g} / \mathrm{ml}$ aprotinin, $2 \mu \mathrm{g} /$ $\mathrm{ml}$ leupeptin, $1 \mu \mathrm{g} / \mathrm{ml}$ pepstatin $\mathrm{A}$, and $1 \%$ phosphatase inhibitor cocktail [Sigma-Aldrich]). Cell lysates were incubated with gentle rocking for 1 hour at $4^{\circ} \mathrm{C}$ and then centrifuged at $12,281 \mathrm{~g}$ for 20 minutes. The supernatant was analyzed as the whole cell lysate. Nuclear extracts were prepared as described previously (62). Extraction of nuclear and cytoplasmic protein fractions were performed using a Nuclear/Cytosol Fractionation Kit (BioVision, K266). 
Western blot analyses were performed using anti-PPP3CA (Santa Cruz Biotechnology Inc., sc-9070 and sc-17808), anti-acetyl-Histone-H3 (Millipore, 06-599), anti-HDAC6 (Cell Signaling Technology, 7558), anti-NFATc1 (Santa Cruz Biotechnology Inc., sc-7294), anti-caspase-3 (Cell Signaling Technology, 9662), anti-cleaved caspase-3 (Cell Signaling Technology, 9661), anti-caspase-7 (Cell Signaling Technology, 9494), anti-cleaved caspase-7 (Cell Signaling Technology, 9491), anti-Bim (Cell Signaling Technology, 2819), antiNF- $\kappa$ B p50 (Santa Cruz Biotechnology Inc., sc-7178), anti-MITF (Abcam, ab12039), anti-HSP70 (Enzo Life Sciences, ADI-SPA-812), anti-FLAG (Sigma-Aldrich, F3165), anti-actin (Sigma-Aldrich, A2066), anti- $\alpha$ tubulin (Covance, MMS-489R), and anti-TATA binding protein (Abcam, ab818) antibodies. Immunoprecipitated proteins were recovered using Protein G-Sepharose 4B Conjugate (Invitrogen). The Luminata Forte HRP Western Substrate (Millipore) was used for chemiluminescent detection, and the images were analyzed with a LAS-1000 (FUJIFILM). Quantitative analyses of bands in analyzed images were performed using Multi Gauge (FUJIFILM). We also quantitated the degree of protein expression change from the blots in Figures 2 and 3. For the immunoprecipitation assays, total cell lysates were prepared as described previously (63), and anti-PPP3CA (Santa Cruz Biotechnology Inc., sc-17808), anti-HSP90 $\alpha$ (Stressgen, ADI-SPA-840), and mouse IgG2b Isotype control (MBL, M077-3) antibodies were used.

$R N A$ extraction, $c D N A$ synthesis, and relative quantification real-time PCR. Total RNA was extracted from patient samples, MM cells, and osteoclasts using TRIzol Reagent (Ambion). cDNA was synthesized from total RNA using a High Capacity cDNA Reverse Transcription Kit (Applied Biosystems). cDNA, TaqMan Gene Expression Master Mix (Applied Biosystems), and TaqMan MGB primer-probe sets (Applied Biosystems) for the target and endogenous control gene were mixed and analyzed on an ABI 7500 Real-Time PCR System or a StepOnePlus Real-Time PCR System (Applied Biosystems). The TaqMan MGB primer-probe sets were as follows: human PPP3CA, Hs00174223_ml; human HDAC6, Hs00195869_ml; mouse PPP3CA, Mm01317678_ml; human $\beta$-actin, human ACTB (Beta Actin) Endogenous Control (Applied Biosystems); and mouse $\beta$-actin, mouse ACTB (Beta Actin) Endogenous Control (Applied Biosystems). Each patient's mRNA amount of PPP3CA divided by that of U266 cells was designated as relative PPP3CA mRNA.

MTT assays. A total of $2 \times 10^{4}$ of the indicated MM cell lines were seeded in a 96-well plate and cultured for 48 hours as indicated. MTT assays were performed using the Cell Counting Kit-8 (DOJINDO) following the manufacturer's instructions, and the plates were read using a Plate CHAMELEON (Hidex). Five independent clones were examined. CD20-positive cells were sorted using CD20 Micro Beads (Miltenyi, 130-091-104) as described in the manufacturer's protocol. A total of $10 \mu \mathrm{g} / \mathrm{ml}$ pokeweed mitogen (PWH) (Sigma-Aldrich) was added to the culture medium to induce the proliferation of CD20-positive cells. In MTT assays of shRNA-transduced U266 or FLAG-vector-transduced KMS-11, a total of $1 \times 10^{4}$ (U266) or $3 \times 10^{4}$ (KMS-11) cells were cultured for 72 hours. The relative viability of $P P P 3 C A$ knockdown or overexpressed clone cells compared with that of control cells is displayed. The viability of each clone and control cells was evaluated by the ratio of the MTT assays value measured after 72 hours culture to that measured just after the cell distribution.

Lentiviral production and transduction. HEK293T cells were transfected with the target plasmid, pMD2.G (Addgene), and psPAX2 (Addgene) by the calcium phosphate transfection method. The culture medium was collected the following day, as well as 2 days later. Lentivirus was concentrated from the collected medium using a Lenti-X Concentrator (Clontech). A mixture of culture medium containing the concentrated lentivirus and $8 \mu \mathrm{g} / \mathrm{ml}$ polybrene was added to the culture medium of the target cells (KMS-11). GFP-positive transduced cells were sorted on a FACSAria Cell Sorter (BD Biosciences). These cells were then cultured under a $2 \mu \mathrm{g} / \mathrm{ml}$ puromycin (Sigma-Aldrich) selection. A nonsilencing-GIPZ lentiviral shRNAmir control (Thermo Scientific) was used as a control for the target plasmid. A human GIPZ lentiviral shRNAmir target gene set (clone ID: KD\#1, V2LHS_235563; KD\#2, V2LHS_131422; and KD\#3, V2LHS_373190)(Thermo Scientific) was used to reduce endogenous PPP3CA. To enhance PPP3CA expression in KMS-11 cells, we constructed a lentiviral expression vector of PPP3CA. FLAG-tagged cDNA from PPP3CA variant 2 (NM_000944) (Kazusa DNA Research Institute) was subcloned into a pcDH1-MSCVMCS-EF1-GFP + puro cDNA Cloning and Expression Vector (System Bioscience, CD713B-1). A lentivirus to induce FLAG- $P P P 3 C A$ was produced, and $P P P 3 C A$-overexpressed clones (clones 1, 2, and 3) were obtained as described previously.

BrdU assays. A total of $5 \times 10^{3}$ KMS-11 cells were seeded in 96-well plates and cultured for 48 hours as indicated. Cell proliferation was analyzed using a Cell Proliferation ELISA, bromodeoxyuridine (BrdU) chemiluminescent Kit (Roche Diagnostics) following the manufacturer's instructions. Plates were read on a 
Plate CHAMELEON (Hidex), and the results were acquired by relative luminescence units (rlu) per second.

Flow cytometric analysis. To examine apoptosis, KMS-11 cells were stained with the ApoAlert Annexin V-FITC Apoptosis Kit (Clontech) following the manufacturer's instructions, and cells were analyzed on an EPICS XL System II Flow Cytometer (Beckman Coulter). Data were analyzed using Kaluza 1.3 (Beckman Coulter).

Xenograft mouse model. Five- to 7-week-old NOD/ShiJic-scid Jcl (NOD/SCID) female mice (CLEA Japan) were used for the in vivo assays. A total of $3 \times 10^{7}$ U266 cells in $100 \mu 1$ of RPMI-1640 were mixed with the same volume of Matrigel (BD Biosciences), and the mixture was s.c. injected into the back of the mouse. Tumors were measured with calipers, and volumes were calculated using the formula $\alpha^{2} \times \beta \times$ 0.5 , where $\alpha$ is the shortest diameter and $\beta$ is the diameter perpendicular to $\alpha$, as described previously (64). When the diameter of the tumor reached $1.0 \mathrm{~cm}$, treatment with vehicle, panobinostat, FK506, or both panobinostat and FK506 was initiated. Panobinostat (provided by Novartis Pharma Inc.) was dissolved in $5 \%$ dextrose (Sigma-Aldrich), and $20 \mathrm{mg} / \mathrm{kg}$ panobinostat was i.p. injected into mice 3 times per week for 3 weeks. FK506 (20\%; provided by Astellas Pharma. Inc.) was dissolved in water, and $10 \mathrm{mg} / \mathrm{kg}$ FK506 was administered orally to mice 3 times per week for 3 weeks. Tumor volume and mice body weight were assessed on alternate days. Mice treated with panobinostat showed body weight loss, and we set $20 \mathrm{mg} / \mathrm{kg}$ panobinostat as the maximum tolerated dose, with less than $15 \%$ body weight loss. After sacrifice, cleavage of caspase-3, PPP3CA expression, and acetylation of histone $\mathrm{H} 3$ in tumors from some mice were examined.

IHC. Tumors from the xenograft mouse model were put in optimal cutting temperature (OCT) compound (Sakura Finetek, 4583) and frozen. The frozen samples were processed as 6- $\mu \mathrm{m}$ sections with cryostat (Leica, CM1850). After fixation and permeabilization, the sections were incubated with anti-cleaved caspase- 3 antibody overnight at $4^{\circ} \mathrm{C}$ centigrade and washed in PBS. The sections were then incubated with anti-rabbit secondary antibody (Vector, MP-7401) for 1 hour at room temperature and washed in PBS. A 3,3'-diaminobenzidine (DAB) reaction was performed using a DAB Peroxidase (HRP) Substrate Kit (Vector, SK-4100) following the manufacture's instructions. Counterstaining by H\&E was also performed.

Induction of mouse BM mononuclear cells into osteoclasts and tartrate-resistant acid phosphatase (TRAP) staining of osteoclasts. Induction of mouse BM mononuclear cells into osteoclasts and TRAP staining of osteoclasts were performed as previously described (65). Mouse BM cells were harvested from the femurs and tibias of female C57 BL/6J mice. They were seeded in 24-well plates and cultured in minimum essential medium $\alpha$ supplemented with $10 \% \mathrm{FCS}, 10 \mathrm{ng} / \mathrm{ml}$ human macrophage colony-stimulating factor (R\&D Systems), penicillin, and streptomycin for differentiation into macrophages. On days 3, 5, and 6, $50 \mathrm{ng} / \mathrm{ml}$ human RANKL (PeproTech) was added to the medium for the induction of macrophages into osteoclasts. We then counted TRAP-positive multinucleated cells (more than 3 nuclei).

Statistics. All data except for those pertaining to the xenograft mouse model are shown as the average \pm SD. Differences between the 2 groups of samples were analyzed using a 1-tailed $t$ test. For differences among the 3 groups of samples, ANOVA using a 1-way layout was performed. When the statistic model proved significant, the differences between combinations of the 2 groups were analyzed using a TukeyKramer test for multiple comparisons. PFS of the patients treated with bortezomib-containing therapies was defined as the duration from the time to start of therapy to that of disease relapse or progression. A Kaplan-Meier analysis was performed for the study of PFS. A $P$ value less than 0.05 was considered significant. Statistics except for those about xenograft mouse model were analyzed with JMP ver.11 (SAS Institute). The time-dependent variations in tumor volume between panobinostat (+)/FK506(-)-treated and panobinostat (+)/FK506(+)-treated mice in a xenograft mouse model were analyzed with SAS ver. 9.4 (SAS Institute) using the GLM procedure. Tumor size difference between 2 groups on designated days were estimated by Scheffe test analyses.

Study approval. In the analyses of patients' samples, all patients signed independent informed consent forms for the sampling and molecular and clinical analyses prior to participation in this study. All clinical investigation was conducted according to Declaration of Helsinki principles. This study was approved by the Institutional Ethics Committees of Tokyo Women's Medical University and Nippon Medical School. All mouse protocols used in this study were approved by the Institutional Animal Care and Use Committee of Tokyo Women's Medical University.

\section{Author contributions}

YI conceived the study, designed and performed the research, collected the patients' samples, analyzed and 
interpreted data, and wrote and edited the manuscript. EO designed and performed the research, analyzed and interpreted data, and wrote and edited the manuscript. ST and SS performed the experiments inducing mouse BM mononuclear cells into osteoclasts. MI and HT collected the patients' samples. YHW conducted the experiments of lentiviral production and transduction. AD performed the immunohistochemical experiments. JT, YM, and TM interpreted data, wrote and edited the manuscript.

\section{Acknowledgments}

We thank M. Shimaoka (Mie University, Tsu, Mie, Japan) for providing scientific input in the preparation of this manuscript; H. Handa (Gunma University, Maebashi, Gunma, Japan) for providing KMS11, KMS-18, and KMS-26MM; and S. Shimizu (Tokyo Women's Medical University, Medical Research Institute) for offering advice about the statistical analyses. This work was supported by a Grant-in-Aid for Scientific Research of the Japan Society for the Promotion of Science, the Takeda Science Foundation, an International Myeloma Foundation Japan Grant, and the Japan Leukemia Research Fund.

Address correspondence to: Yoichi Imai, Department of Hematology, Tokyo Women's Medical University, 8-1 Kawada-cho, Shinjuku-ku, Tokyo 162-8666, Japan. Phone: 81.3.3353.8111; E-mail: imaiyo@twmu.ac.jp.

1. Richardson PG, et al. PANORAMA 2: panobinostat in combination with bortezomib and dexamethasone in patients with relapsed and bortezomib-refractory myeloma. Blood. 2013;122(14):2331-2337.

2. San-Miguel JF, et al. Panobinostat plus bortezomib and dexamethasone versus placebo plus bortezomib and dexamethasone in patients with relapsed or relapsed and refractory multiple myeloma: a multicenter, randomised, double-blind phase 3 trial. Lancet Oncol. 2014;15(11):1195-1206.

3. Hideshima T, et al. Small-molecule inhibition of proteasome and aggresome function induces synergistic antitumor activity in multiple myeloma. Proc Natl Acad Sci U S A. 2005;102(24):8567-8572.

4. Hideshima T, Richardson PG, Anderson KC. Mechanism of action of proteasome inhibitors and deacetylase inhibitors and the biological basis of synergy in multiple myeloma. Mol Cancer Ther. 2011;10(11):2034-2042.

5. Catley L, et al. Aggresome induction by proteasome inhibitor bortezomib and alpha-tubulin hyperacetylation by tubulin deacetylase (TDAC) inhibitor LBH589 are synergistic in myeloma cells. Blood. 2006;108(10):3441-3449.

6. Nawrocki ST, et al. Aggresome disruption: a novel strategy to enhance bortezomib-induced apoptosis in pancreatic cancer cells Cancer Res. 2006;66(7):3773-3781.

7. Heider U, et al. Synergistic interaction of the histone deacetylase inhibitor SAHA with the proteasome inhibitor bortezomib in mantle cell lymphoma. Eur J Haematol. 2008;80(2):133-142.

8. Santo L, et al. Preclinical activity, pharmacodynamic, and pharmacokinetic properties of a selective HDAC6 inhibitor, ACY1215, in combination with bortezomib in multiple myeloma. Blood. 2012;119(11):2579-2589.

9. Hui KF, Chiang AK. Combination of proteasome and class I HDAC inhibitors induces apoptosis of NPC cells through an HDAC6-independent ER stress-induced mechanism. Int J Cancer. 2014;135(12):2950-2961.

10. Gachet S, Ghysdael J. Calcineurin/NFAT signaling in lymphoid malignancies. Gen Physiol Biophys. 2009;28(spec no focus):F47-F54.

11. Medyouf $\mathrm{H}$, et al. Targeting calcineurin activation as a therapeutic strategy for T-cell acute lymphoblastic leukemia. Nat Med. 2007;13(6):736-741.

12. Bhattacharyya S, et al. NFATc1 affects mouse splenic B cell function by controlling the calcineurin — NFAT signaling network JExp Med. 2011;208(4):823-839.

13. Pham LV, Tamayo AT, Yoshimura LC, Lin-Lee YC, Ford RJ. Constitutive NF- $\mathrm{B}$ and NFAT activation in aggressive B-cell lymphomas synergistically activates the CD154 gene and maintains lymphoma cell survival. Blood. 2005;106(12):3940-3947.

14. Fu L, Lin-Lee YC, Pham LV, Tamayo A, Yoshimura L, Ford RJ. Constitutive NF- $\mathrm{kB}$ and NFAT activation leads to stimulation of the BLyS survival pathway in aggressive B-cell lymphomas. Blood. 2006;107(11):4540-4548.

15. West AC, Johnstone RW. New and emerging HDAC inhibitors for cancer treatment. J Clin Invest. 2014;124(1):30-39.

16. Choudhary $\mathrm{C}$, et al. Lysine acetylation targets protein complexes and co-regulates major cellular functions. Science. 2009;325(5942):834-840.

17. Mandawat A, et al. Pan-histone deacetylase inhibitor panobinostat depletes CXCR4 levels and signaling and exerts synergistic antimyeloid activity in combination with CXCR4 antagonists. Blood. 2010;116(24):5306-5315.

18. Bots $\mathrm{M}$, et al. Differentiation therapy for the treatment of $\mathrm{t}(8 ; 21)$ acute myeloid leukemia using histone deacetylase inhibitors. Blood. 2014;123(9):1341-1352.

19. Carrasco DR, et al. High-resolution genomic profiles define distinct clinico-pathogenetic subgroups of multiple myeloma patients. Cancer Cell. 2006;9(4):313-325.

20. Agnelli L, et al. Molecular classification of multiple myeloma: a distinct transcriptional profile characterizes patients expressing CCND1 and negative for 14q32 translocations. J Clin Oncol. 2005;23(29):7296-7306.

21. Durie BG, Salmon SE. A clinical staging system for multiple myeloma. Correlation of measured myeloma cell mass with presenting clinical features, response to treatment, and survival. Cancer. 1975;36(3):842-854.

22. Palumbo A, et al. Revised International Staging System for Multiple Myeloma: A Report From International Myeloma Working Group. J Clin Oncol. 2015;33(26):2863-2869.

23. Noborio-Hatano K, et al. Bortezomib overcomes cell-adhesion-mediated drug resistance through downregulation of VLA-4 expression in multiple myeloma. Oncogene. 2009;28(2):231-242. 
24. Someren JS, Faber LE, Klein JD, Tumlin JA. Heat shock proteins 70 and 90 increase calcineurin activity in vitro through calmodulin-dependent and independent mechanisms. Biochem Biophys Res Commun. 1999;260(3):619-625.

25. Kupatt C, et al. Heat shock protein 90 transfection reduces ischemia-reperfusion-induced myocardial dysfunction via reciprocal endothelial NO synthase serine 1177 phosphorylation and threonine 495 dephosphorylation. Arterioscler Thromb Vasc Biol. 2004;24(8):1435-1441.

26. New M, Olzscha H, La Thangue NB. HDAC inhibitor-based therapies: can we interpret the code? Mol Oncol. 2012;6(6):637-656.

27. Kovacs JJ, et al. HDAC6 regulates Hsp90 acetylation and chaperone-dependent activation of glucocorticoid receptor. $\mathrm{Mol}$ Cell. 2005;18(5):601-607.

28. Richardson PG, Mitsiades CS, Laubach JP, Lonial S, Chanan-Khan AA, Anderson KC. Inhibition of heat shock protein 90 (HSP90) as a therapeutic strategy for the treatment of myeloma and other cancers. Br J Haematol. 2011;152(4):367-379.

29. Neckers L, Workman P. Hsp90 molecular chaperone inhibitors: are we there yet? Clin Cancer Res. 2012;18(1):64-76.

30. Hemenway CS, Heitman J. Calcineurin. Structure, function, and inhibition. Cell Biochem Biophys. 1999;30(1):115-151.

31. Brewin J, et al. Generation of EBV-specific cytotoxic T cells that are resistant to calcineurin inhibitors for the treatment of posttransplantation lymphoproliferative disease. Blood. 2009;114(23):4792-4803.

32. Fonseca et al. Clinical and biologic implications of recurrent genomic aberrations in myeloma. Blood. 2003;101(11):4569-4575.

33. Grand EK, Chase AJ, Heath C, Rahemtulla, Cross NCP. Targeting FGFR3 in multiple myeloma: inhibition of t(4;14)-positive cells by SU5402 and PD173074. Leukemia. 2004;18(5): 962-966.

34. Otsuki T, et al. Expression of fibroblast growth factor and FGF-receptor family genes in human myeloma cells, including lines possessing $\mathrm{t}(4 ; 14)(\mathrm{q} 16.3 ; \mathrm{q} 32.3)$ and FGFR3 translocation. Int J Oncol. 1999;15(6):1205-1212.

35. Lombardi L, et al. Molecular characterization of human multiple myeloma cell lines by integrative genomics: insights into the biology of the disease. Genes Chromosomes Cancer. 2007;46(3):226-238.

36. Chen S, et al. A Bim-targeting strategy overcomes adaptive bortezomib resistance in myeloma through a novel link between autophagy and apoptosis. Blood. 2014;124(17):2687-2697.

37. Buchholz M, Ellenrieder V. An emerging role for $\mathrm{Ca}^{2+} /$ calcineurin/NFAT signaling in cancerogenesis. Cell Cycle. 2007;6(1):16-19.

38. Trushin SA, Pennington KN, Algeciras-Schimnich A, Paya CV. Protein kinase C and calcineurin synergize to activate I $\mathrm{B}$ kinase and NF-кB in T lymphocytes. J Biol Chem. 1999;274(33):22923-22931.

39. Keats JJ, et al. Promiscuous mutations activate the noncanonical NF-kB pathway in multiple myeloma. Cancer Cell. 2007;12(2):131-144.

40. Nishioka C, et al. Histone deacetylase inhibitors induce growth arrest and apoptosis of HTLV-1-infected T-cells via blockade of signaling by nuclear factor $\kappa B$. Leuk Res. 2008;32(2):287-296.

41. Kuhn DJ, et al. Potent activity of carfilzomib, a novel, irreversible inhibitor of the ubiquitin-proteasome pathway, against preclinical models of multiple myeloma. Blood. 2007;110(9):3281-3290.

42. Abe M, et al. Osteoclasts enhance myeloma cell growth and survival via cell-cell contact: a vicious cycle between bone destruction and myeloma expansion. Blood. 2004;104(8):2484-2491.

43. Zawawi MS, Dharmapatni AA, Cantley MD, McHugh KP, Haynes DR, Crotti TN. Regulation of ITAM adaptor molecules and their receptors by inhibition of calcineurin-NFAT signalling during late stage osteoclast differentiation. Biochem Biophys Res Commun. 2012;427(2):404-409.

44. Chai RC, et al. Molecular stress-inducing compounds increase osteoclast formation in a heat shock factor 1 protein-dependent manner. J Biol Chem. 2014; 289(19):13602-13614.

45. Zhang BW, et al. T cell responses in calcineurin A $\alpha$-deficient mice. J Exp Med. 1996;183(2):413-420.

46. Wang D, Iclozan C, Liu C, Xia C, Anasetti C, Yu XZ. LBH589 enhances T cell activation in vivo and accelerates Graft-versusHost Disease in Mice. Biol Blood Marrow Transplant. 2012;18(8):1182-1190.

47. Noll E, et al. Myeloma plasma cells alter the bone marrow microenvironment by stimulating the proliferation of mesenchymal stromal cells. Haematologica. 2014;99(1):163-171.

48. Kino T, et al. FK-506, a novel immunosuppressant isolated from a Streptomyces. J Antibiot (Tokyo). 1987;40(9):1249-1255.

49. Afshar-Sterle S, et al. Fas ligand-mediated immune surveillance by T cells is essential for the control of spontaneous B cell lymphomas. Nat Med. 2014;20(3):283-290.

50. Michigami T, et al. Cell-cell contact between marrow stromal cells and myeloma cells via VCAM-1 and $\alpha 4 \beta 1$-integrin enhances production of osteoclast-stimulating activity. Blood. 2000;96(5):1953-1960.

51. Haggarty SJ, Koeller KM, Wong JC, Grozinger CM, Schreiber SL. Domain-selective small-molecule inhibitor of histone deacetylase 6 (HDAC6)-mediated tubulin deacetylation. Proc Natl Acad Sci U S A. 2003;100(8):4389-4394.

52. Laubach JP, Moreau P, San-Miguel JF, Richardson PG. Panobinostat for the treatment of multiple myeloma. Clin Cancer Res. 2015;21(21):4767-4773.

53. Maiso P, et al. The histone deacetylase inhibitor LBH589 is a potent antimyeloma agent that overcomes drug resistance. Cancer Res. 2006;66(11):5781-5789.

54. Cheng J, Tang W, Su Z, Wei Q. Mutation of calcineurin subunit B M118 influences the activities of NF-AT and p53, but not calcineurin expression level. Biochem Biophys Res Commun. 2011;413(3):481-486.

55. Auner HW, et al. Reduced intensity-conditioned allogeneic stem cell transplantation for multiple myeloma relapsing or progressing after autologous transplantation: a study by the European Group for Blood and Marrow Transplantation. Bone Marrow Transplant. 2013;48(11):1395-1400.

56. Oerlemans R, et al. Molecular basis of bortezomib resistance: proteasome subunit $\beta 5$ (PSMB5) gene mutation and overexpression of PSMB5 protein. Blood. 2008;112(6):2489-2499.

57. Zhu YX, et al. RNAi screen of the druggable genome identifies modulators of proteasome inhibitor sensitivity in myeloma including CDK5. Blood. 2011;117(14):3847-3857.

58. Xu D, et al. D111/Notch activation contributes to bortezomib resistance by upregulating CYP1A1 in multiple myeloma. Biochem Biophys Res Commun. 2012;428(4):518-524.

59. Mulligan G, et al. Gene expression profiling and correlation with outcome in clinical trials of the proteasome inhibitor bortezomib. Blood. 2007;109(8):3177-3188. 
60. Breitkreutz I, et al. Lenalidomide inhibits osteoclastogenesis, survival factors and bone-remodeling markers in multiple myeloma. Leukemia. 2008;22(10):1925-1932.

61. Ocio EM, et al. In vitro and in vivo rationale for the triple combination of panobinostat (LBH589) and dexamethasone with either bortezomib or lenalidomide in multiple myeloma. Haematologica. 2010;95(5):794-803.

62. Yokoyama A, et al. Leukemia proto-oncoprotein MLL forms a SET1-like histone methyltransferase complex with menin to regulate Hox gene expression. Mol Cell Biol. 2004;24(13):5639-5649.

63. Qu X, et al. Arsenic trioxide exerts antimyeloma effects by inhibiting activity in the cytoplasmic substrates of histone deacetylase 6. PLoS One. 2012;7(2):e32215.

64. Catalano MG, et al. Cytotoxic activity of the histone deacetylase inhibitor panobinostat (LBH589) in anaplastic thyroid cancer in vitro and in vivo. Int J Cancer. 2012;130(3):694-704.

65. Sato S, et al. Central control of bone remodeling by neuromedin U. Nat Med. 2007;13(10):1234-1240 